\title{
A simplified ALE model for finite element simulation of ballistic impacts with bullet splash - development and experimental validation
}

\author{
Riccardo Andreotti \\ Callens ${ }^{\circledR}$ AREA3 Via Merini 3721100 Varese, Italy \\ Dipartimento di Meccanica, Politecnico di Milano, via La Masa 1, 20156 Milano, Italy \\ riccardo.andreotti@callens.it
}

Sergio Abate

Reparto Investigazioni Scientifiche, RIS-Roma, viale Tor di Quinto 151, 00191 Roma, Italy sergio.abate@carabinieri.it

\section{Andrea Casaroli}

Dipartimento di Meccanica, Politecnico di Milano, via La Masa 1, 20156 Milano, Italy

andrea.casaroli@polimi.it

Mauro Quercia

Callens ${ }^{\circledR}$ AREA3 Via Merini 3721100 Varese, Italy

mauro.quercia@area3.it

Riccardo Fossati

RTM Breda, Via Po 84, 20032 Cormano (MI), Italy

riccardo.fossati@rtmbreda.it

\section{Marco V. Boniardi}

Dipartimento di Meccanica, Politecnico di Milano, via La Masa 1, 20156 Milano, Italy marco.boniardi@polimi.it

ABSTRACT. An original simplified finite element model is proposed to simulate the effects of non-penetrating ballistic impacts causing the so-called bullet splash phenomenon (complete bullet fragmentation), while no fragmentation is caused to the target. The model is based on the Arbitrary Lagrangian Eulerian formulation (ALE) and it simulates the impact as a fluidstructure interaction. The bullet splash phenomenon has been tested by experimental analyses of AISI 304L plates impacted by 9x21 FMJ (full metal jacket) bullets. The model has been developed with the aim of creating a simplified approach to be used in the industry and forensic sciences to simulate the non-penetrating interaction of soft impactors with hard targets. Comparisons between evidence and simulation results lead to the conclusion

\section{OPEN ACCESS}

Citation: Andreotti R., Abate S., Casaroli A., Quercia M., Fossati R., Boniardi M.V., A simplified ALE model for finite element simulation of ballistic impacts with bullet splash - development and experimental validation, Frattura ed Integrità Strutturale, $\mathrm{xx}$ (2021) 223-245.

Received: 01.06.2021

Accepted: 10.06.2021

Published: 01.07.2021 
that the proposed approach can be used in a conservative way to estimate both local and global effects of bullet-splash phenomena.

KEYWORDS. Ballistic impact; Bullet splash; Stainless steel plate; Arbitrary Lagrangian Eulerian (ALE); Finite element simulation (FEM); Explicit solver.
Copyright: (C) 2021 This is an open access article under the terms of the CC-BY 4.0, which permits unrestricted use, distribution, and reproduction in any medium, provided the original author and source are credited.

\section{INTRODUCTION}

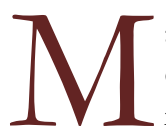

any applications, particularly in aerospace and defense industry, involve the assessment of the structural consequences of impacts such as bird strike (Heimbs, 2012 [1]), hail strike (Anghileri et al., 2005 [2]), or ballistic impacts against structures, with the aim of optimizing their response in terms of protective capabilities. In industry, the optimization of protective capabilities of a structural system is mainly done by means of finite element simulations.

This paper aims at proposing and discussing the effectiveness of an easy-to-use model for finite element simulations in the field of terminal ballistics for industrial and forensic applications, conceived for the assessment of protective structures impacted by splashing bullets.

The typical bullet splash phenomenon [3] was brilliantly captured by photographer Harold Edgerton in 1938 [4], and is due to the low stiffness and strength of the impactor compared to the strength, stiffness, and inertia of the target, causing the high fragmentation of the bullet and the deflection of its fragment's trajectory. Bullet splash is what generally happens when the bullet hits the target at impact angles near to 90 degrees and is unable either to penetrate nor to cause fragmentation of the target; the bullet itself, instead, undergoes large deformations and eventually a complete fragmentation into small debris, which are subsequently deflected and projected around the epicenter of the impact by the target surface.

This phenomenon is relevant for industrial applications in the field of protective functionality of structures because it represents what generally happens when a solid protective structure works properly, being able to locally withstand the pressure field caused by the impact and maintaining its continuity. Consequently, the impact forces locally generated are transmitted to the surrounding structures. In this framework, the optimization of an effective protective structural system thus passes through the verification of the response of the overall system to a vast range of impact tests in all the possible locations at various incidence angles, to assure that the entire structure can safely withstand all the possible impact scenarios, not only locally, but globally.

The possibility of simulating a vast range of load cases in a consistent and stable manner is nowadays fundamental to save time and costs in the process of developing a structural system with multiple safety requirements. But developing finite element simulations in the field of protective functionalities against ballistic impacts is still a challenging problem for the industry, with high costs due to the material characterization and model development and validation.

Most of the literature available on ballistic impacts simulation is focused on analyzing and simulating the effects of rigid projectiles impacting on deformable targets, with the aim of estimating the ballistic limit velocity and the modes of failure of the target. Rajput \& Iqbal (2017) [5] showed both experimentally and numerically the effects of rigid projectiles of different nose shapes impacting on thin aluminum plates at different speed. In 2014, Yunfei et al. [6] demonstrated and simulated the effectiveness of impactors of different strengths and deformability on penetrating single and multilayered metallic plates. Early studies about numerical strategies for the simulation of three-dimensional fragmentation phenomena during impacts, involving simplified geometries and brittle materials, has been carried out by Camacho \& Ortiz in 1996 [7]. Pandolfi and Ortiz (2002) [8] were able to reproduce the dynamic crack propagation of notched specimens during three point bend dynamic tests and their work was taken as a reference for a more recent study by Bresciani et al. (2016) [9] on the simulation of the interaction between projectile and target involving the fragmentation of both, using the adaptive solid mesh to SPH technique.

No studies have been found focused on the analysis and simulation of soft bullets interacting with much harder targets causing the bullets-splash phenomenon, which is also common in the forensic investigation of crime scenes and is typically observed in the aerospace and defense industry when a hard solid protective structure is subjected to non-penetrating impacts.

To investigate the phenomenon, we propose the experimental analysis of AISI 304 steel plates (4mm thick) impacted by 9x $21 \mathrm{~mm}$ FMJ bullets, causing the typical bullets-splash phenomena at different incidence angle. The analysis of the residual deformation of the plates, the local state of hardening around the impact area, and the evidence of complete bullet 
fragmentation has been used to conceive and validate a simplified simulation model that, under the given assumptions, does not require any complex characterization of the impactor and can be implemented easily on all the most popular finite element explicit simulation platforms commercially available.

The model is based on the observation that during the bullet splash phenomena the change in shape of the impactor and its subsequent fragmentation makes the impactor act like a fluid mass rather than a proper solid. The modelling approach has been inspired from the simulation techniques commonly used for the numerical assessment of airframe structures against bird-impacts (Heimbs, 2012 [1]). In fact, the softness of the 9x21 mm FMJ bullet, compared to the target, makes the observed phenomena qualitatively comparable with what happens during bird-strikes: the compact heterogeneous mass of skin, flesh, bones, and biological fluids hits a much harder surface at severe speed, encountering a complete loss of integrity. The high fragmentation of the impactor causes then the mass of debris to act like a fluid flow deflected by the target surface. Bird Strike is the most frequent cause of accident in aviation, and the industry has developed standard tests to assess the safety of the aircraft and its occupants against this risk. Since the early 90 s modern aerospace engineering procedures include the use of simulation to predict the response of the airframe structures under bird-strike tests to optimize the structural design in advance. The scientific literature on the modelling of the impactor for bird-strike simulation (Heimbs, 2012) shows that the models commonly used to simulate bird strikes use few different formulations, all having in common the extreme simplification of the impactor, always represented as a homogeneous volume of soft or pre-fragmented matter, characterized by an initial simplified geometry to represent the main dimensions of the real impactor, associated with equivalent mass and speed (Heimbs, 2012 [1]).

This paper introduces a similar strategy to simplify the bullet and simulate ballistic impacts once the hardness of the impactor is significantly lower than the one of the target. Even though 9x21FMJ bullets are conceptually very simple, a rigorous characterization and modelling of their mechanical behavior during the impacts would have been extremely difficult and unpractical for industrial use. Therefore, to model the phenomenon, a simplified approach has been hypothesized, based on the assumption that the structural effects of these impacts on the target are mostly due to the inertial forces needed to deviate the mass of the bullet debris from their initial trajectory, and the internal forces needed to stretch and break the impactor during the interaction with the target can be considered neglectable. This strong assumption allowed us to avoid the characterization and modelling of the deviatoric component of the constitutive laws governing the bullet's behavior, therefore modelling it like a volume of compressible inviscid fluid with initial shape, linear compressibility, mass and velocity being the only few physical parameters to mechanically describe the impactor.

To implement these hypotheses into a simulation that can be of practical use in the industry, we chose to model the interaction between impactor and target using the arbitrary Lagrangian-Eulerian formulation (ALE) [10], which, during the last two decades, has been successfully used for several industrial applications to investigate the mechanical interaction between soft and hard continua like fluid-structure interaction, bird-strike [11], and impacts involving soft materials [12], guaranteeing strong stability of the calculation even in case of extremely large deformation fields.

This technique, despite some intrinsic and well-known drawbacks [1], minimizes the risk of local instabilities, which are particularly negative inconveniences when encountered in the industry, where engineers frequently need to process several batch analyses with multiple load cases and sensitivity tests on tight deadlines.

Section 2 of the article illustrates the experimental evidence collected to quantify the effects of the bullet-splash phenomena used to develop and validate the model. The effects of the impacts are quantified in terms of microhardness distribution in the impact area and residual deformation of the plates depending on the impact angle. Section 3 introduces the simulation model and the main phases of its development. In section 4 the simulation results are discussed and validated based on the comparison with experimental data in terms of equivalent plastic strain and residual deformation of the plates. To compare the microhardness measurements taken on the impact area of the samples with the equivalent plastic strain field estimated by the simulations, an empirical linear relation by Qiao et al. [13] was applied. A discussion on the accuracy of the results and some practical indications to overcome the drawbacks of the technique are then introduced to conclude the section, with the aim of giving the reader a practical guide to the use of the model always in a conservative way, depending on the scope.

In section 5 we summarize the conclusions and discuss the possible improvements and developments to the research.

\section{EXPERIMENTAL ANALYSIS OF THE BULLET SPLASH PHENOMENON}

$B$

ullet splash is an expression coming from the terminal ballistics and legal jargon (Oxford University Press, 2020) [14] defined as "the particles sprayed from a bullet on its impact against metal or other hard material". To verify and measure the effects of bullet splash on the target, we tested the response of 4-millimeter AISI 304 plates impacted 
by 9x21 FMJ bullets, with the scope of guaranteeing the non-penetration of the bullet and a significant ductile response of the plate. This way the field of plastic strain and plastic deformation of the plates have been treated as a measure of the severeness of the impacts. The visual analysis of the impact point and a debris collection screen allowed the investigation of the bullet fragmentation.

\section{Ballistic test procedures}

The ballistic tests were conducted at the experimental shooting range of the Reparto Investigazioni Scientifiche (RIS) of Carabinieri in Rome (Fig. 1). The bullets were shot by an automatic handgun Beretta Storm D PX4 actuated by a pneumatic shooting machine which allows the control and reproducibility of the shots in terms of position and direction (Fig. 2). The projectile speed was measured by means of a magnetic speed detector (Fig. 3) positioned at $2.5 \mathrm{~m}$ from the muzzle of the gun to avoid the detonation gases to interfere with the measurements. The samples were positioned 14.3 meters far from the shooting machine according to the shooting area scheme represented in Fig. 4. The speed measured was consistently 320-330 meters per second.

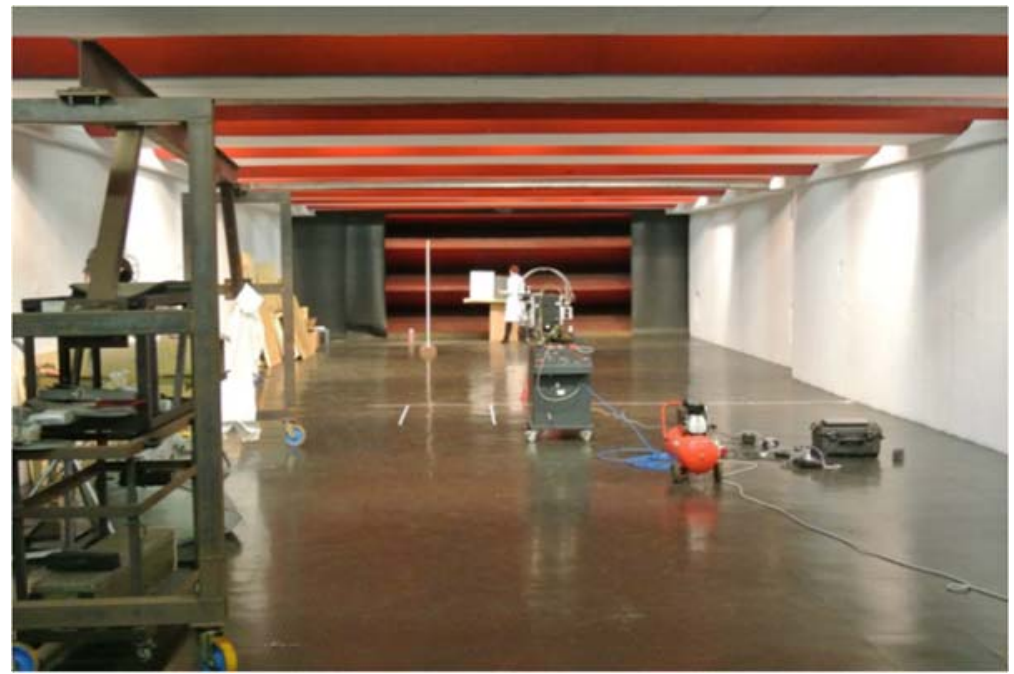

Figure 1: The experimental shooting range of the Reparto Investigazioni Scientifiche (RIS) of Carabinieri in Rome, Italy, 2016.

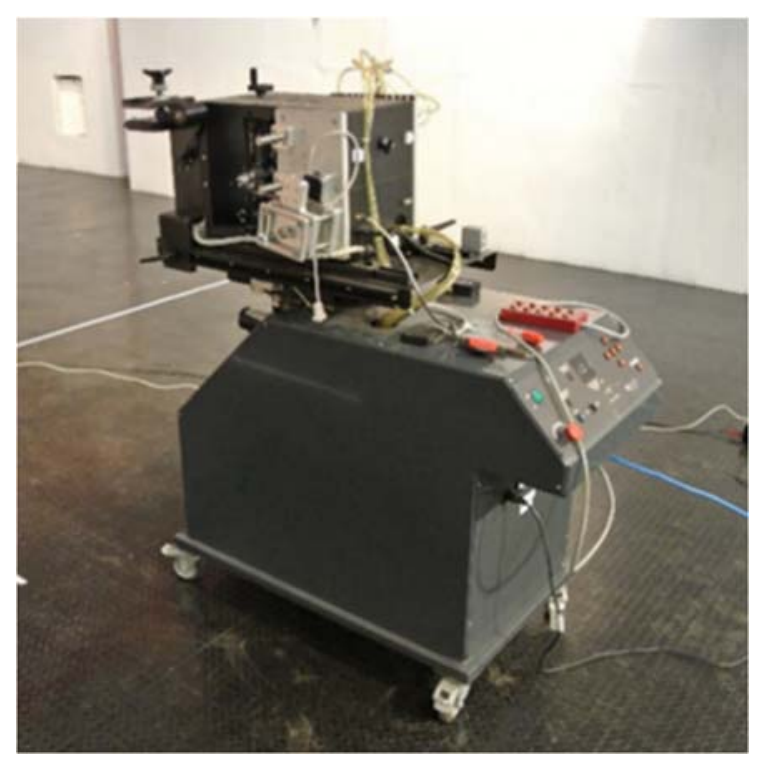

Figure 2: The pneumatic shooting machine allowing the control and reproducibility of the shots.

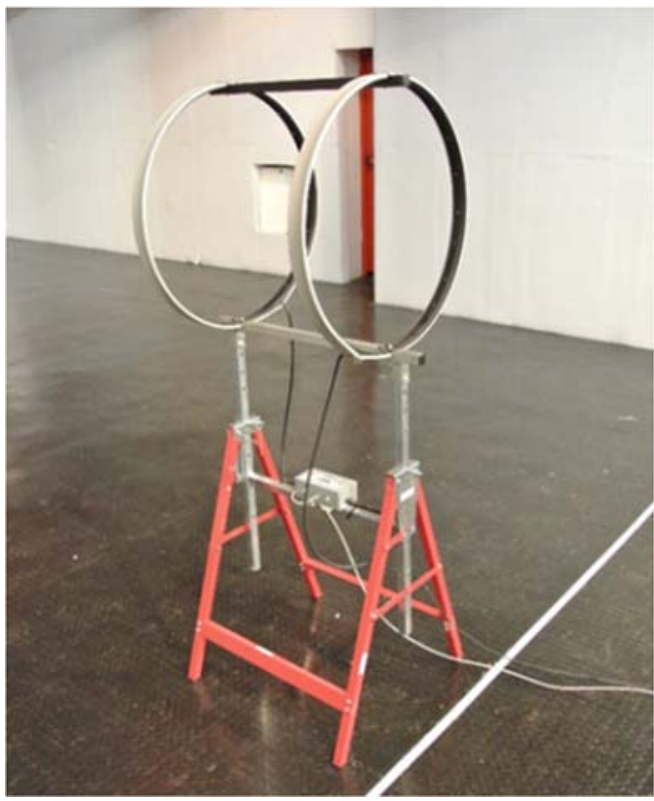

Figure 3: The magnetic speed detector used to control the projectile speed at $2.5 \mathrm{~m}$ from the gun muzzle. 


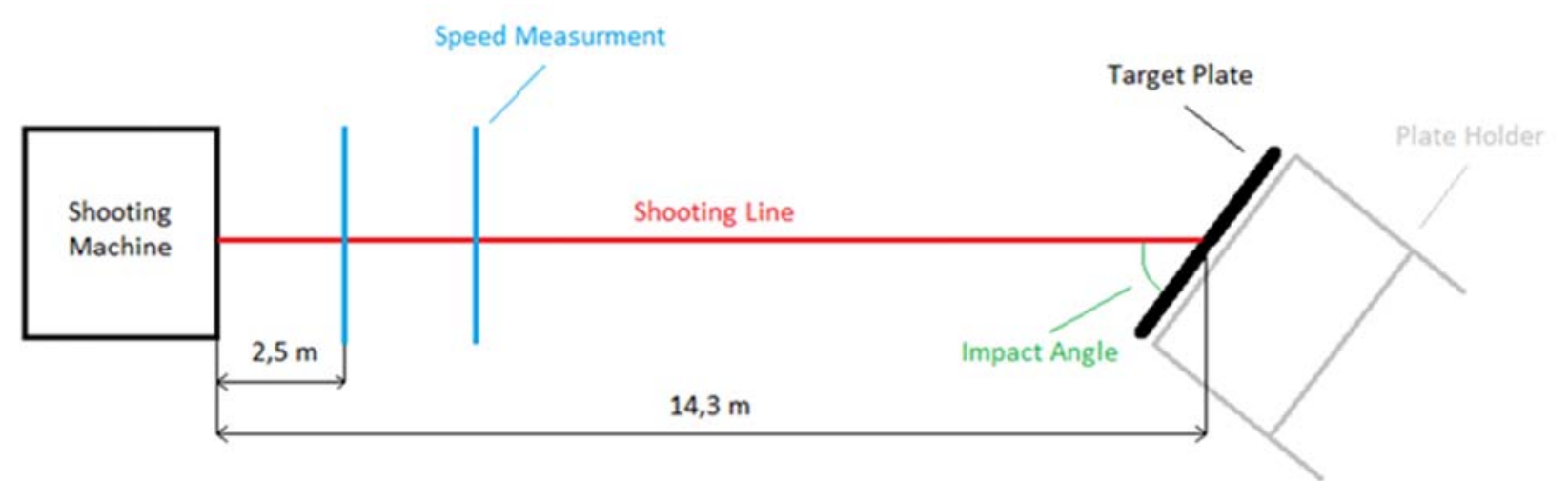

Figure 4: General scheme of the shooting area.

\section{Target samples positioning}

The tested samples were $4 \mathrm{~mm} \times 500 \mathrm{~mm} \times 500 \mathrm{~mm}$ plates, kept in position by means of a plate holder to which they were clamped as shown in Fig. 5.

The 4mm thick AISI 304 plates were chosen to guarantee that the targets would stand the impacts without allowing any penetration or fragmentation, being able to collect enough plastic strain to allow to experimentally catch the residual deformation of the plates as well as the local hardening in the impact areas.

A polystyrene witness screen to collect bullets debris was also positioned sideways depending on the orientation of the sample with respect to the shooting machine. The angular position of the sample was changed manually by the operator.

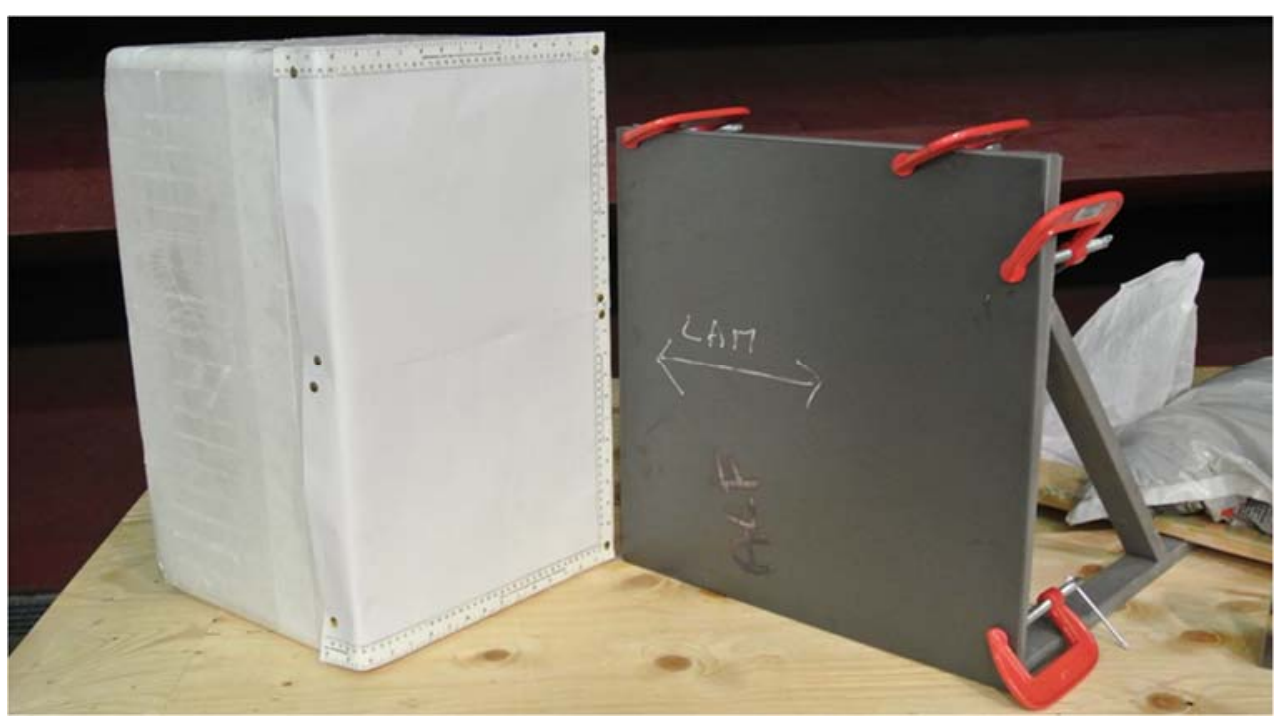

Figure 5: The tested samples plates ( $4 \mathrm{~mm} \times 500 \mathrm{~mm} \times 500 \mathrm{~mm})$, kept in position by means of a support frame to which they were clamped at the angles. Sideways to the sample plate a polystyrene screen was positioned in order to collect debris.

\section{Chemical characterization of AISI 304L inox steel}

The tested samples were made of austenitic stainless steel X2 Cr Ni 18-9, commercially known as AISI 304L, where "L" stands for "Low-carbon", which improves the weldability and ductility i.e. formability and high energy absorption under impulsive loading conditions (toughness). The exact composition of the alloy was verified quantometer measurements, whose results are resumed in Tab. 1.

\begin{tabular}{llllllllll}
\hline $\mathrm{C}$ & $\mathrm{Si}$ & $\mathrm{Mn}$ & $\mathrm{P}$ & $\mathrm{S}$ & $\mathrm{Cr}$ & $\mathrm{Ni}$ & $\mathrm{Mo}$ & $\mathrm{Cu}$ & $\mathrm{Fe}$ \\
0.023 & 0.351 & 1.46 & 0.0291 & 0.0017 & 18.11 & 8.11 & 0.25 & 0.46 & 70.809 \\
\hline
\end{tabular}

Table 1: Chemical composition (weight \%) of X2 Cr Ni 18-9 samples. 
Quasi-static tensile characterization of AISI 304L plates

To test the effective static strength of the material, two tensile samples were cut using a waterjet machine according to the UNI EN ISO 6892-1-2009 standard (see Fig. 6) and tested. This characterization work on the undeformed material is useful for allowing both the numerical characterization of the material and significant comparisons between pre and post impact properties. The engineering stress strain curve of the AISI 304L is shown in Fig. 7; the standard mechanical characterization is resumed in Tab. 2.

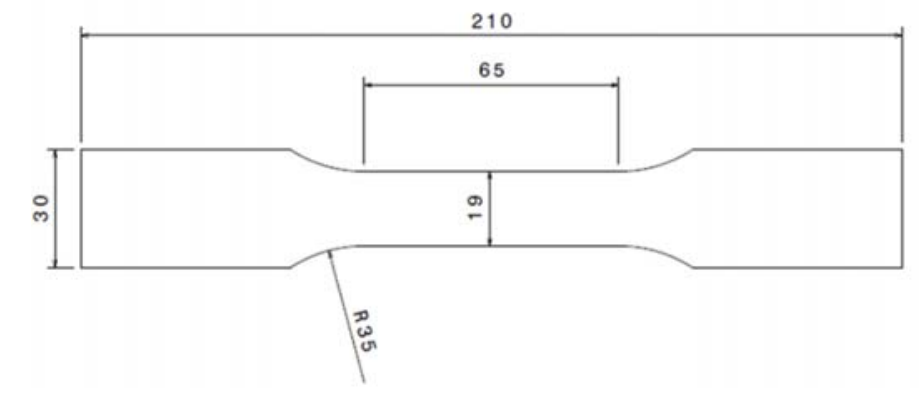

Figure 6: Tensile characterization sample design according to UNI EN ISO 6892-1-2009 standard.

\begin{tabular}{ll}
\hline Material & X2 Cr Ni 18-9 \\
E $[\mathrm{MPa}]$ & 205000 \\
$\mathrm{Rp} 0,2[\mathrm{MPa}]$ & 297 \\
$\mathrm{Rm}[\mathrm{MPa}]$ & 673 \\
$\mathrm{~A}[\%]$ & 63 \\
$\mathrm{Z}[\%]$ & 61 \\
\hline
\end{tabular}

Table 2: Mechanical proprieties of AISI 304L.

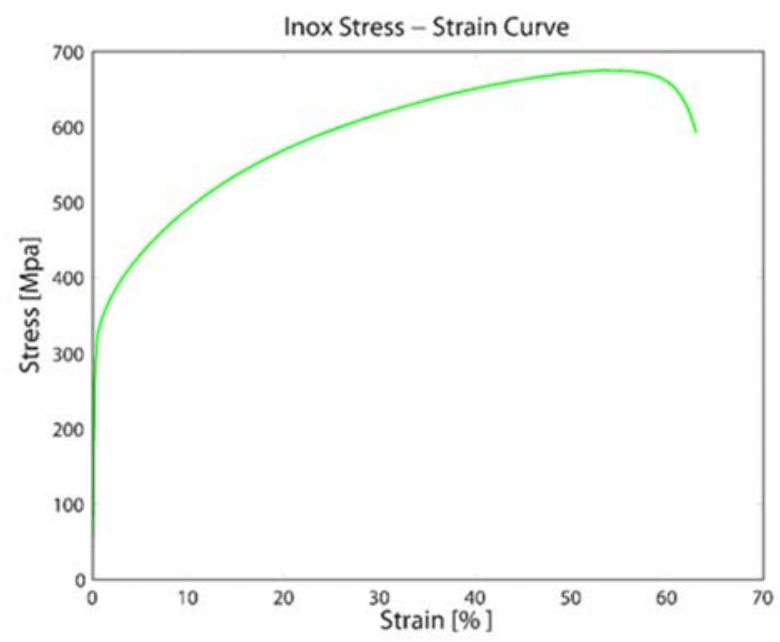

Figure 7: AISI 304L's engineering stress - strain curve.

\section{Ammunition and projectile}

The impactor of 9x21 FMJ cartridges is composed by a core, made of lead, and the thin brass jacket, aimed to prevent the erosion of the soft core with a thickness of around $0.04 \mathrm{~mm}$. The mass of the bullet is $8 \mathrm{~g}$ and the expected velocity is between $320-330 \mathrm{~m} / \mathrm{s}$. 


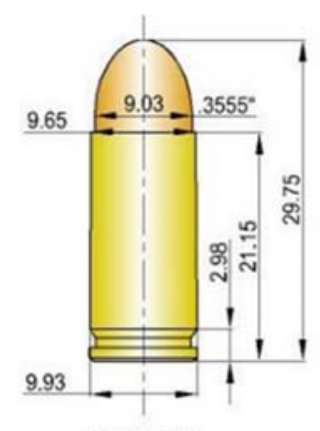

$9 \times 21 \mathrm{IMI}$

Figure 8: 9x21 mm FMJ scheme (length scale in millimeters). The ball length is $15 \mathrm{~mm}$.

\section{Experimental results}

Each 500x500mm plate was shot four times, one time for each quarter, thanks to the laser pointing system of the shooting machine. The large dimensions of the plates compared to the expected extension of the local effects of each single shot allowed us to exclude any interference among different shot-altered areas.

The AISI 304L plates were tested at four different incidence angles $\left(90^{\circ}, 85^{\circ}, 60^{\circ}, 45^{\circ}\right)$ with 2 replicates.

As expected, none of the tested samples show any sign of penetration. The observation of all different samples shows how the projectile has been almost completely pulverized because of the impacts, especially at higher angles. Traces of the bullet debris are clearly visible, with the epicenter characterized by the significant presence of residuals of the projectile (the brown/orange brass jacket of the bullet is particularly evident) accumulated in the curved surface of the plates. Fig. 9 shows the aspect of the samples impacted at different angles impact angle.

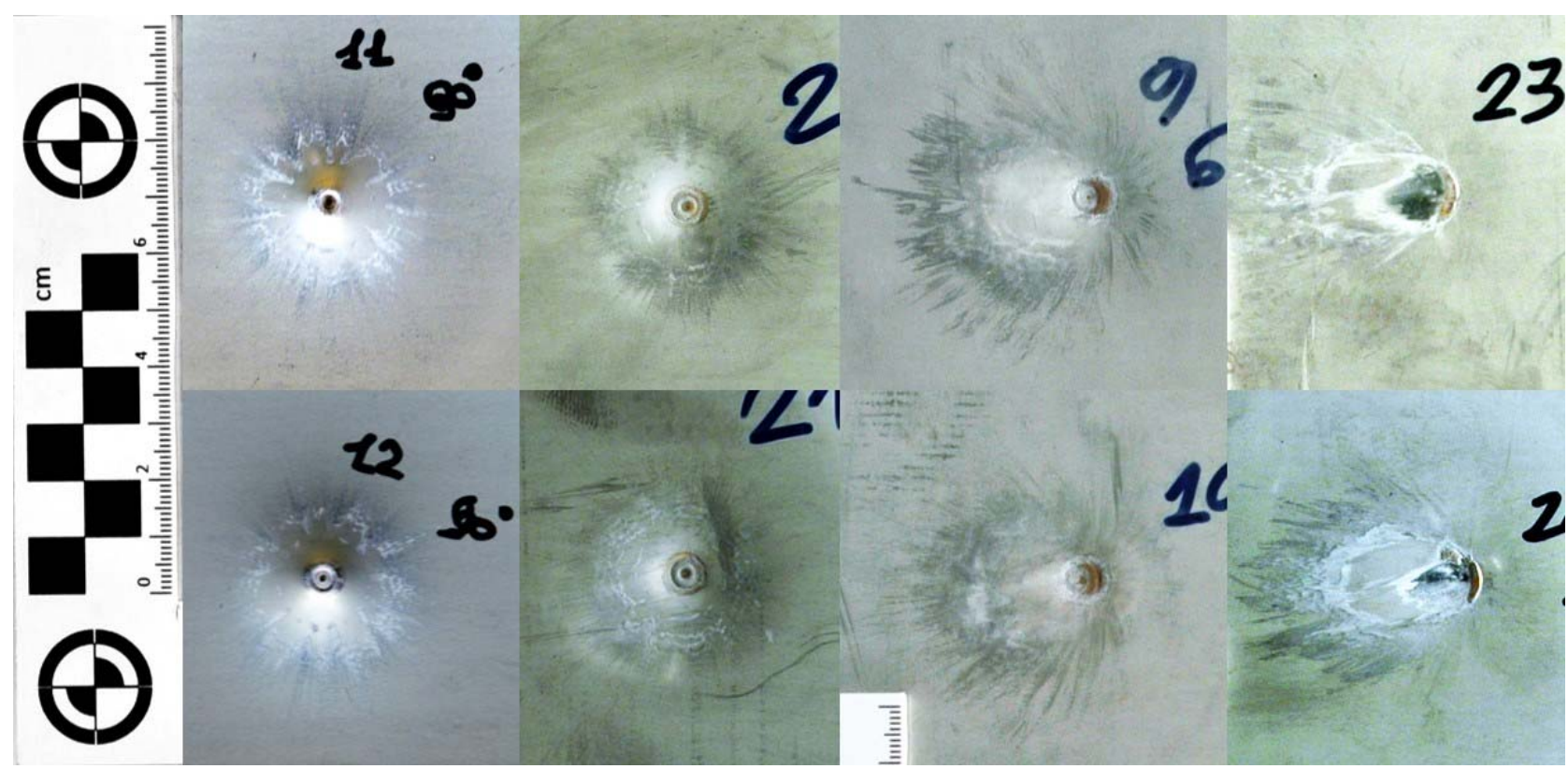

Figure 9: Impacted surface of the eight samples tested: left to right the four impact angle values: $90^{\circ}, 85^{\circ}, 60^{\circ}, 45^{\circ}$. The two rows represent the two replicates of the shots.

According to the evidence available from the debris collection screen positioned sideways to the plates, the rebound angle of the debris is practically neglectable compared to the incidence angle. All the samples show traces of the debris (Fig. 9), clearly visible around the epicenter of the impact, for a radial distance of about 50 millimeters. The epicenter shows significant presence of residuals of the projectile accumulated in the curved surface of the plates. It is also evident how the angle affects the deflection of the debris. The number of visible traces is around $10^{2}$.

The measurement has been carried out using a Borletti mechanical comparator (with a sensibility of 10 micron) and a calibrated workbench. The results are collected in Fig. 10. 


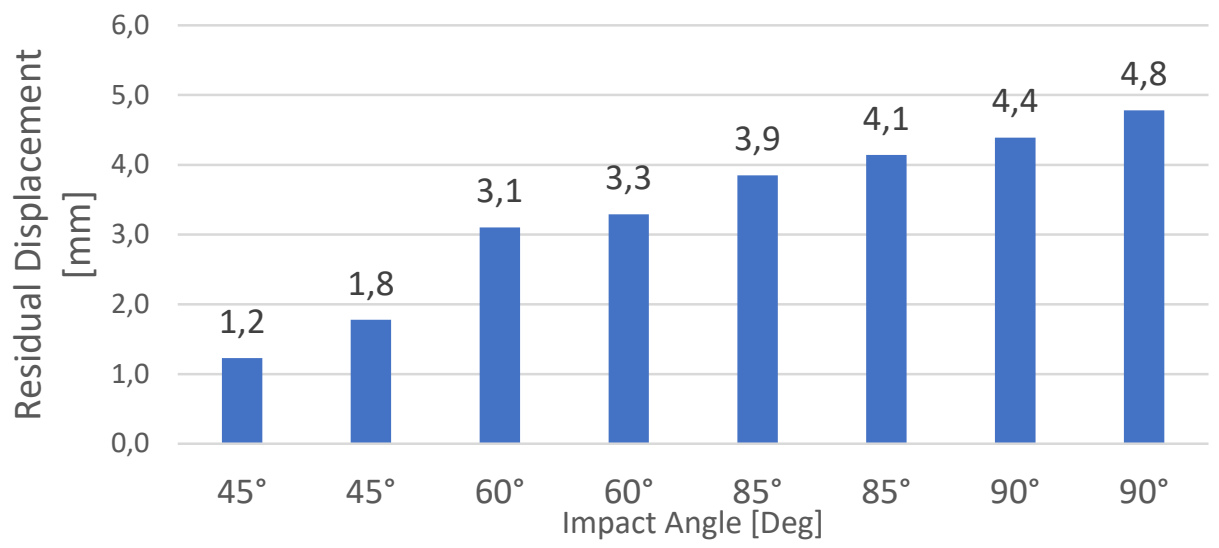

Figure 10: Maximum back-plate residual displacements in millimeters.

\section{Micrographic Analyses}

Micrographic analyses were conducted to verify the microstructural condition of the permanently deformed plates near the impacts epicenter. For this characterization work, the samples were sectioned to check the cross section of the projectiletarget interaction zone, the cut was performed along the lamination direction.

The micrographic analysis (Fig. 12) shows that neither signs of penetration nor cracks are present in the section of the impacted samples. They also show no evident grain size or shape changes (Fig. 14). No strain concentration zones can be observed, but a smooth and continuous deformation field. These results confirm the visual examination and the insufficient penetration capability of the chosen projectile associated with the $4 \mathrm{~mm}$-thick AISI 304L plate under investigation.

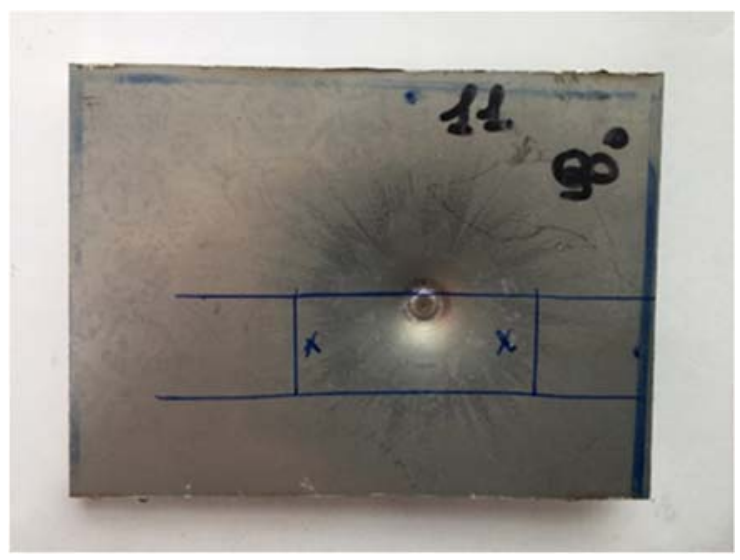

Figure 11: Sample cut for micrographic analysis.

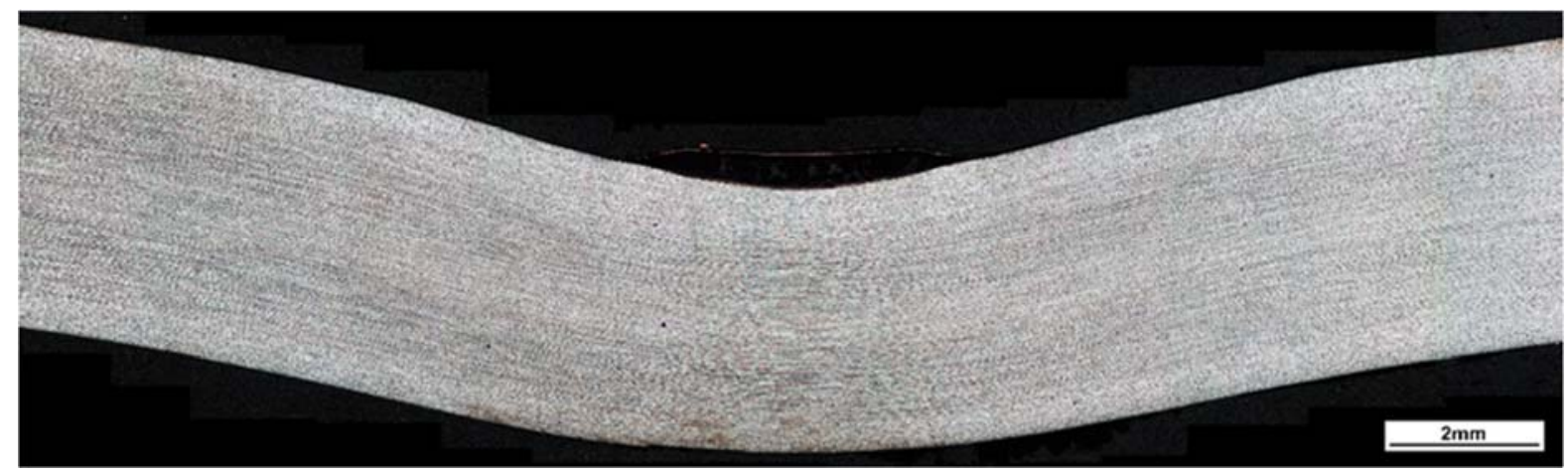

Figure 12: $90^{\circ}$ (50x magnification). The deformation field appears smooth all along the section without any relevant spall phenomenon even at the epicenter. The epicenter shows the residue of the brass jacket of the projectile. 


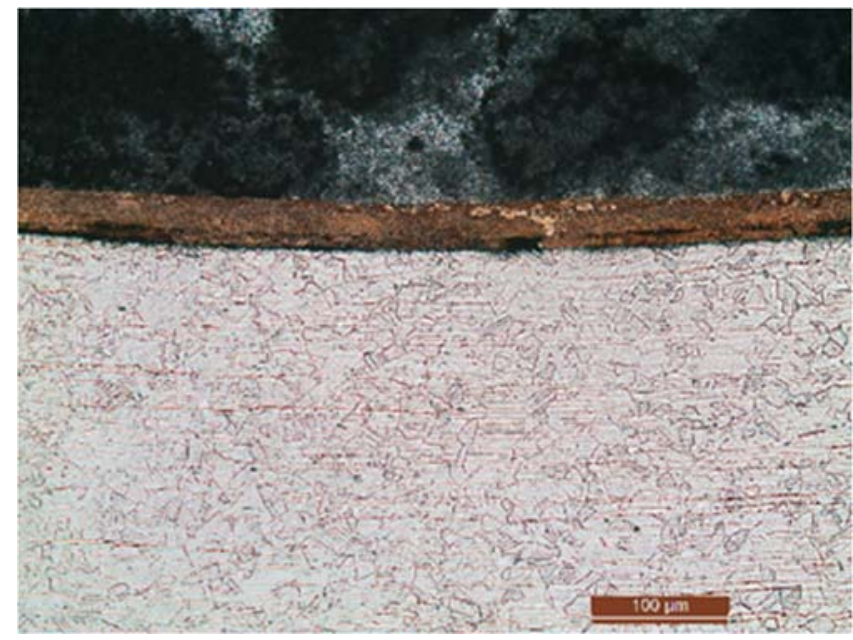

Figure 13: $90^{\circ}$ (200x magnification). At the epicenter, the residue of the brass jacket of the projectile is clearly visible.

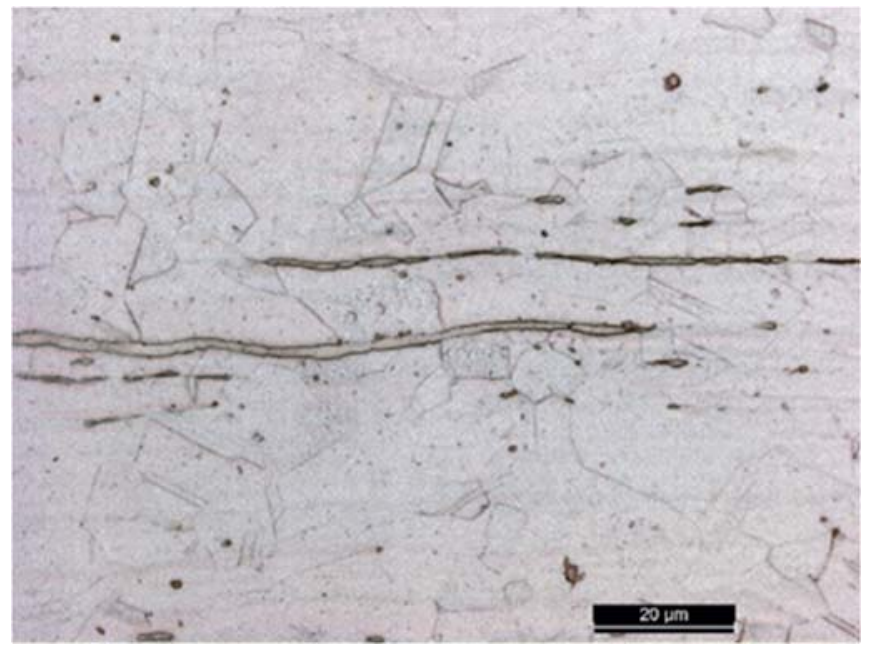

Figure 14: $90^{\circ}$ (1000x magnification). The grain size and shape at the epicenter of the impact does not show any variation compared to the original state of the material.

\section{Micro-hardness Analyses}

Micro-hardness measurements were performed along the section of the plate across the impact zone; this can be considered an indicator of the amount of plastic strain collected by the samples. Hardness is an empirical and punctual measure of the mechanical properties of the material and its value grows with the amount of plastic deformation locally collected by the hardening material. We defined a path all along the impact zone (Fig. 15), at 200 micrometres depth from the impact surface to avoid the boundary effect and conducted forty measures along the path. Five control measures were also taken in the undeformed zone (Fig. 15).

The micro-hardness profile of the 90-degrees impacted sample (Fig. 16) is characterized by two peaks well over 300HV which are almost symmetrical with respect to the local minimum at $270 \mathrm{HV}$, located in correspondence to the epicentre of the impact. The two peaks are 1.7 millimetres far from the epicentre. At farther distances from the epicentre the HV profile decreases gradually until the HV level reaches the values of the undeformed plate, which happens at around 15 millimetres far from the epicentre of the impact. Analog HV profile characterizes the plate impacted at 85 degrees, with two peaks at around $300 \mathrm{HV}$ and a local minimum at the epicentre (Fig. 17). The HV profiles of the plates impacted at 60- and 45-degrees angle do not show evident peaks but a maximum value located at the epicentre (Fig. 18 and Fig. 19). It is evident how the peaks of hardness decrease as impact angle decreases. 


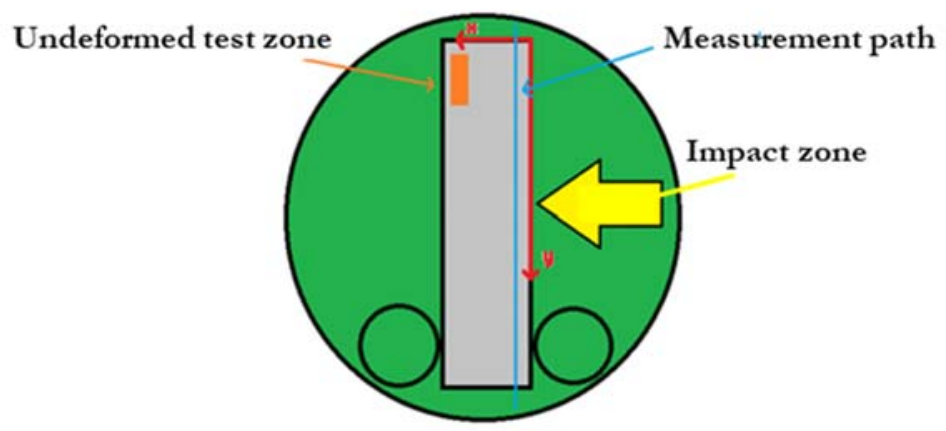

Figure 15: Path (blue line) all along which the micro hardness measures were taken. Undeformed zone is marked orange.

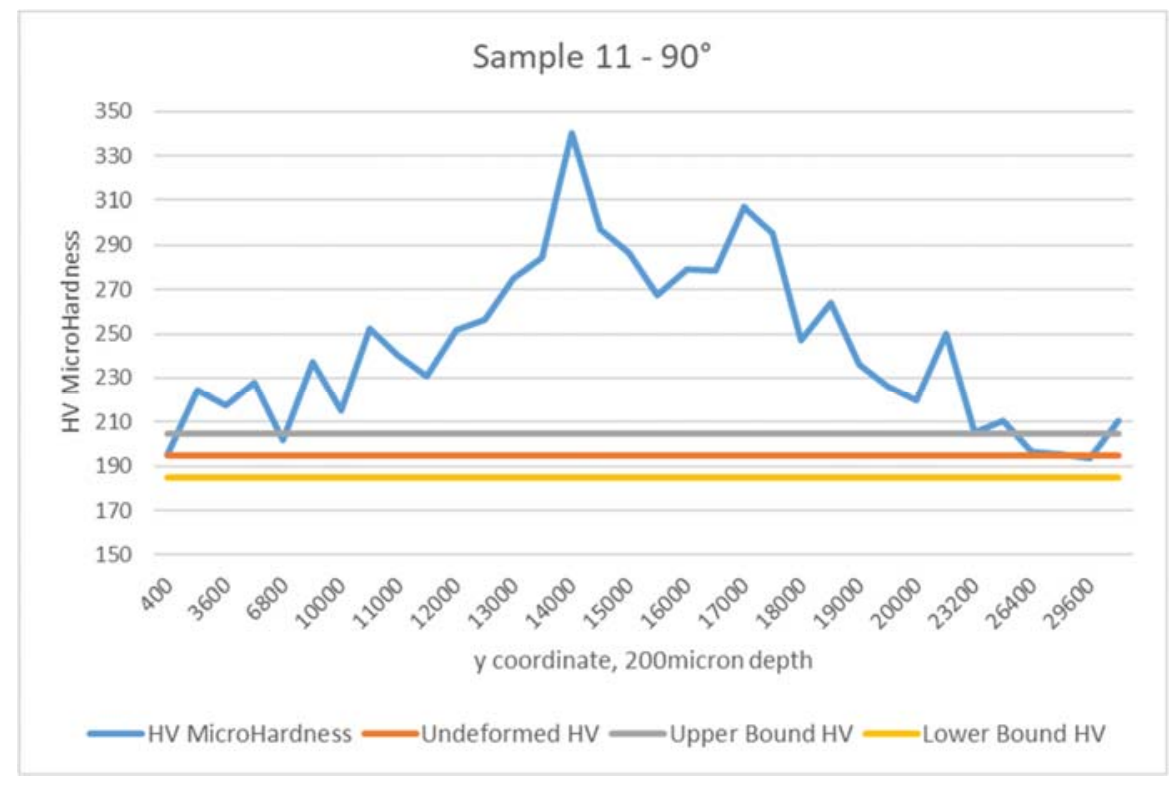

Figure 16: Micro-hardness profile for $90^{\circ}$ impacts. Two peaks well over $300 \mathrm{HV}$ are located symmetrically with respect to the relative minimum at $270 \mathrm{HV}$, corresponding to the epicentre of the impact. These features give the HV distribution an M-shaped profile.

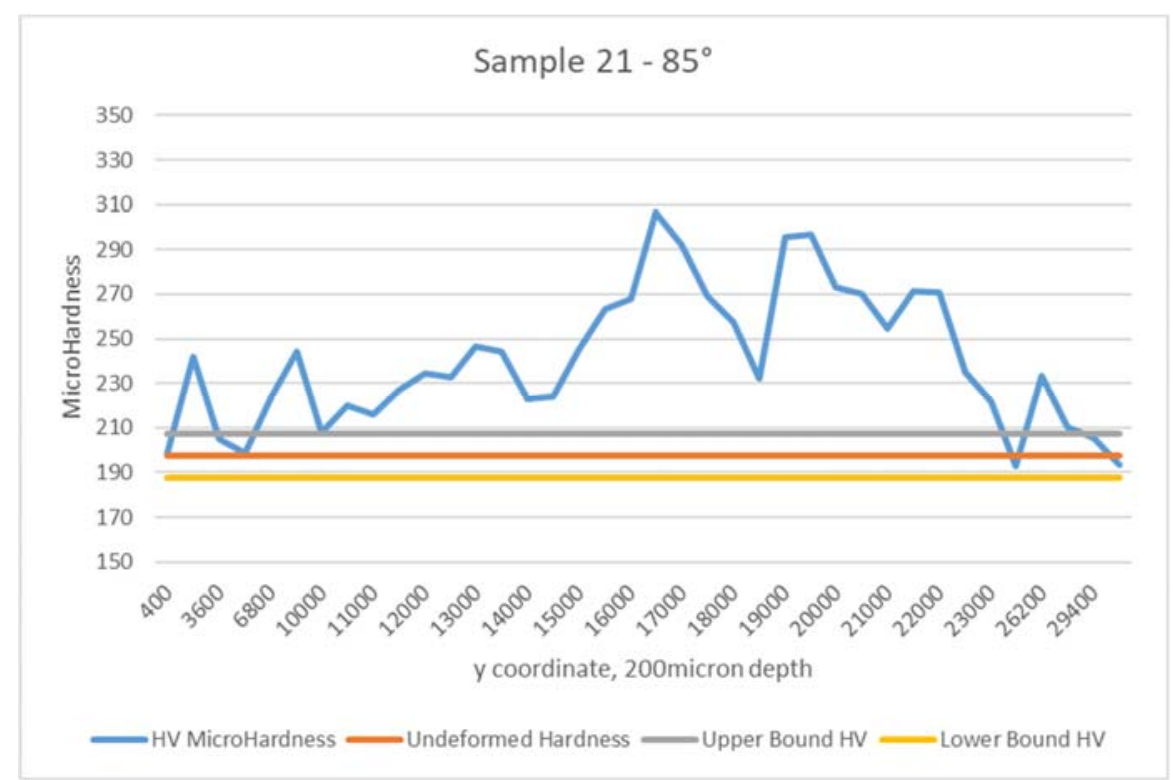

Figure 17: Micro-hardness profile for $85^{\circ}$ impacts. Two peaks at around $300 \mathrm{HV}$ are located symmetrically with respect to the relative minimum at $230 \mathrm{HV}$, corresponding to the epicentre of the impact. These features give the HV distribution an M-shaped profile. 


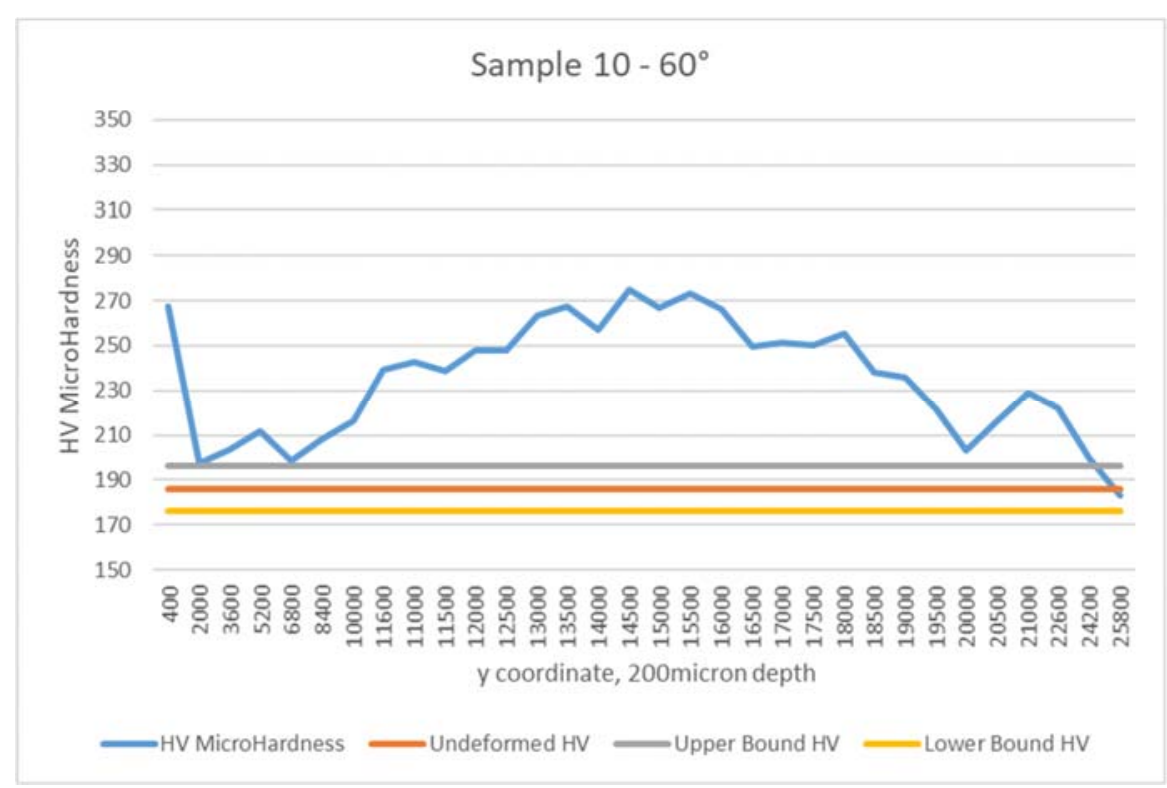

Figure 18: Micro-hardness profile for $60^{\circ}$ impacts. No significant peaks are measured. The maximum value is $275 \mathrm{HV}$, located at the epicentre of the impact.

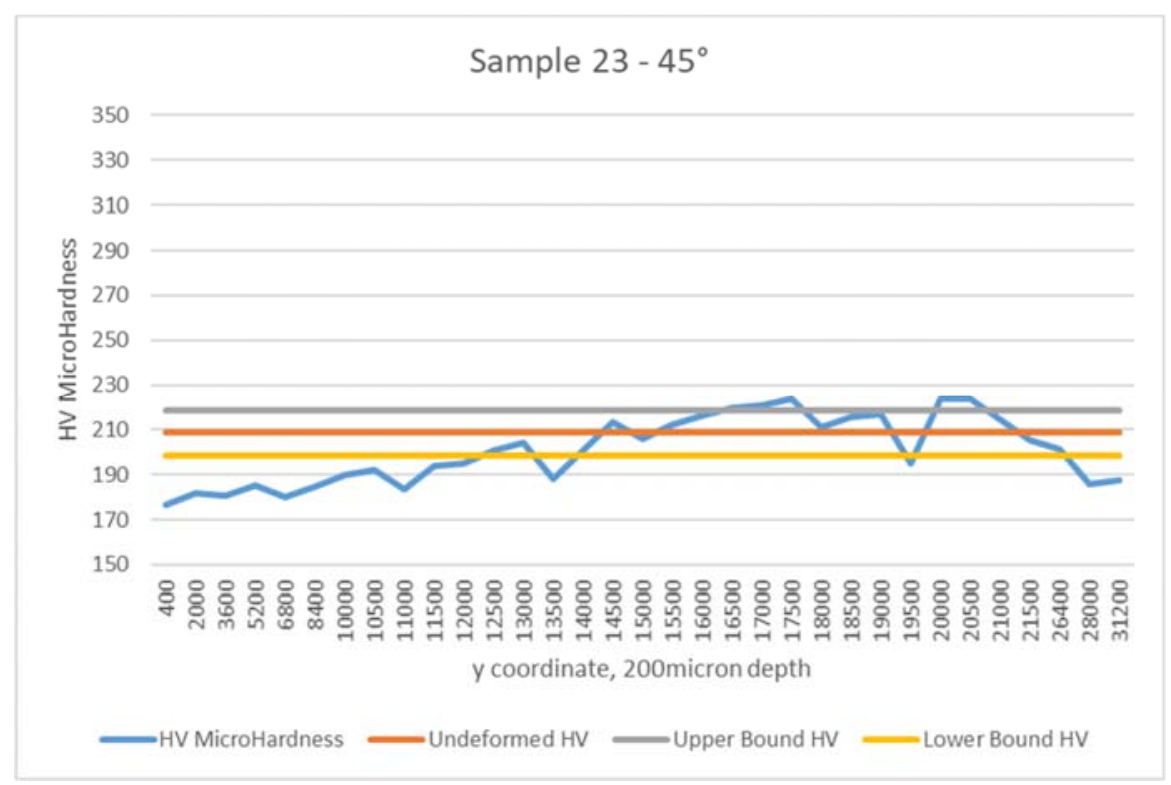

Figure 19: Micro-hardness profile for $45^{\circ}$ impacts. No significant peaks are observed. The maximum value is $224 \mathrm{HV}$, located at the epicentre of the impact.

\section{DEVELOPMENT OF THE NUMERICAL MODEL}

$\checkmark$ he numerical models were developed according to the experimental evidence, characterized by high fragmentation of the bullet and a reasonably continuous field of plastic strain of the target as demonstrated by the micro-hardness measures. The simulations were conducted by means of the well-established explicit solver LS-DYNA.

A simplified finite element model for the splashing impactor

The bullet is defined as a volume fraction of an 50x50x40 millimeter Eulerian material reference system. The position and geometry of the impactor is initialized by means of a shell container representing the external faces of the bullet. The portion 
of the eulerian mesh internal to the container is initialized with the material associated with the bullet, while the external volume is associated with void (Fig. 20).

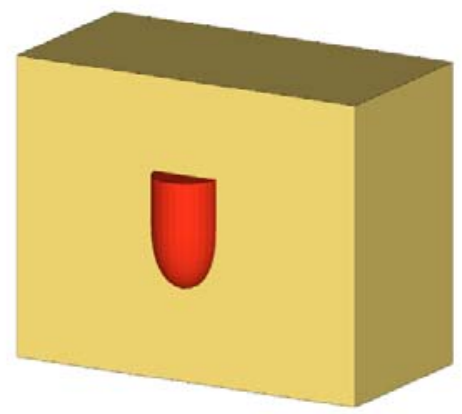

Figure 20: Initial void mesh (yellow) and bullet geometry container (red) to initialize the position and shape of the impactor with ALE formulation.

The ALE formulation allows the entire eulerian reference system to follow the center of mass of the bullet. The interaction between the impactor and the target is guaranteed by means of a penalty algorithm [10] (Fig. 21).

The material associated with the bullet is a homogeneous inviscid fluid defined as *MAT_NULL [15] with a density value $\varrho_{0}=10090 \mathrm{~kg} / \mathrm{m}^{3}$ to guarantee that the total mass associated to the bullet would equal the actual mass of the impactor $(8 \mathrm{~g})$. The compressibility of the fluid is regulated by a linear polynomial equation of state (*EOS_LINEAR_POLYNOMIAL [15]) with all the coefficients being associated with zero value except for the elastic bulk modulus imposed equal to $\beta=45.8$ GPa, a value representative of solid lead [16]. So that the pressure $p$ depends linearly on the variation of density as stated in Eqn. (1).

$$
p=\beta\left(\frac{\rho}{\rho_{0}}-1\right)
$$

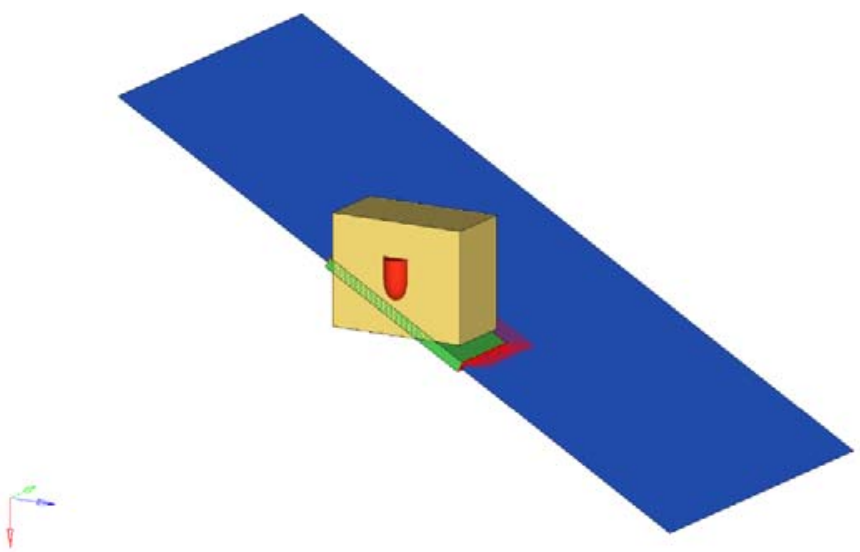

Figure 21: $60^{\circ}$ Impact. Arbitrary Lagrangian Eulerian interaction between bullet (red) and solid mesh plate (green).

\section{Finite element model of the plate}

The plates have been discretized as a 3D structured finite elements mesh, which motion field is described according to a Lagrangian specification.

Being interested in the detailed analysis of the through-thickness stress and strain fields in the surroundings of the impact zone, we opted for a local model characterized by a highly structured 3D solid mesh composed by 6-nodes fully integrated hexahedral elements with homogeneous size, extended to include the entire impactor-target interaction zone $(60 \mathrm{~mm} \mathrm{x}$ $60 \mathrm{~mm}$ extension). The rest of the plate was instead simplified with shell elements. The structural continuity of the plate was then ensured by rigid connections between solid nodes and the corresponding shell nodes.

To reduce the computational cost, a symmetry plane boundary condition was associated with the plane containing the bullet velocity direction and the direction normal to the sample surface. The shell plate boundary nodes are also constrained in the direction normal to the plate (Fig. 22). 


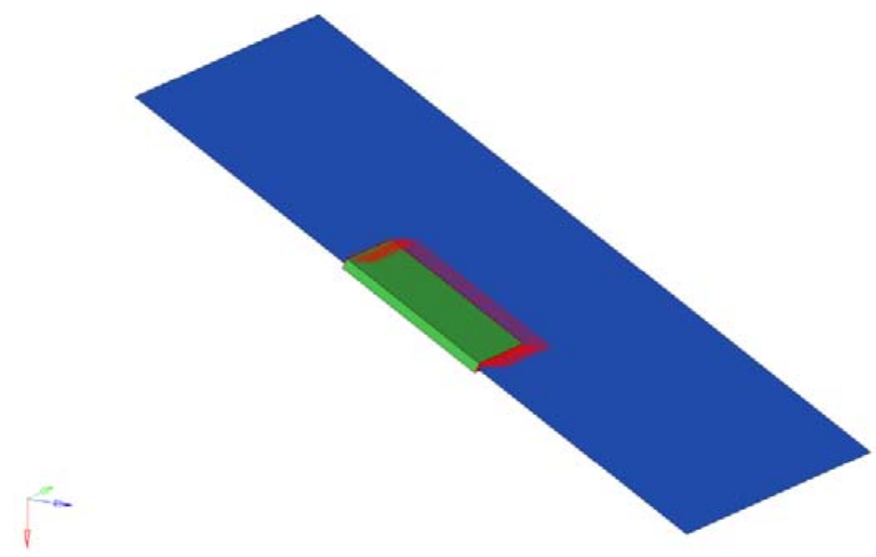

Figure 22: 3D representation of the finite element model of the plate. The blue surface represents the shell mesh, in green is represented the 3D solid mesh. Red marks represent the rigid link to connect shell nodes to solid nodes at the interface between shell and solid meshes.

\section{Constitutive model associated with AISI 304L}

The plates are associated with an elastic plastic material model implementing rate effect and damage (*MAT_PLASTICITY_WITH_DAMAGE [15]). The static parameters of the model are set as: density $\varrho=7800 \mathrm{~kg} / \mathrm{m}^{3}$, Young Modulus E $=200000 \mathrm{MPa}$, Poisson Ratio $\nu=0.33$, Hardening Modulus ETAN $=1508.4 \mathrm{MPa}$, Damage Initiation Strain EPPF $=68.8 \%$, Numerical Strain at Break EPPFR $=140 \%$ (for mesh size $0.2 \mathrm{~mm}$ ).

The rate effect is implemented by means of the two empirical constants $(\mathrm{D}, \mathrm{q})$ that parameterize the Cowper-Symonds model (Eqn. 2) that introduces the rate effect as a dimensionless coefficient acting on the static yield stress of the material $\left(\sigma_{0}\right)$ scaling it to the corresponding value $(\sigma)$ at a generic strain-rate $\varepsilon$. The values of the parameters $\mathrm{D}=100 / \mathrm{s}$ and $\mathrm{q}=10$ were taken from literature [17].

$$
\sigma=\sigma_{0}\left[1+\left(\frac{\dot{\varepsilon}}{D}\right)^{1 / q}\right]
$$

\section{Convergency of the equivalent plastic strain field with increasing nodal density}

The mesh size of the 3D solid elements representing the interaction zone of the plate was set at 0.2 millimeters after a sensitivity study of the equivalent plastic strain peaks estimated with 90-degree impacts simulated with models of increasing nodal density (Fig. 23). The sensitivity study showed a stabilization of the peak of equivalent plastic strain at the value of 0.4 with a nodal density of 5 nodes per millimeter. The impactor mesh size was then set to 0.5 millimeters to guarantee the convergence of the penalty contact between impactor and target.

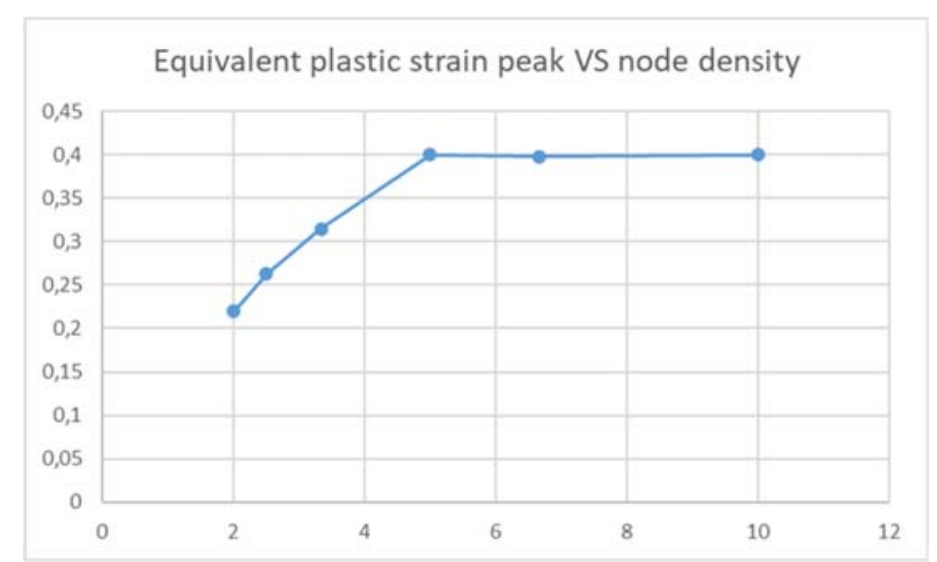

Figure 23: Sensitivity of the peak of equivalent plastic strain (ordinate) calculated with increasing nodal density (abscissa in nodes/mm). 


\section{NUMERICAL SIMULATION RESULTS AND VALIDATION}

o verify the effectiveness of the model in predicting the effects of the impacts on the target, the simulation results have been compared to the experimental evidence in terms of maximum residual displacement and plastic strain. The plastic strain field collected by the real samples have been calculated from the micro-hardness profiles of the samples thanks to an empirical linear correlation (eq.3) between plastic strain and microhardness established by Qiao et al. [13] for AISI 304L:

$$
H V_{304 L}=382 \times \varepsilon_{p}+152
$$

from which, by reversing the correlation:

$$
\varepsilon_{p}=\frac{H V_{304 L}-152}{382}
$$

where $\varepsilon_{\mathrm{p}}$ is the plastic strain and $\mathrm{HV}_{304 \mathrm{~L}}$ is the measure of Vickers micro-hardness.

\section{0-degree impact}

The simulation of the 90-degree impact shows the progressive deformation of the impactor (Fig. 24: ), with the mass of fluid representing the debris of the bullet flowing radially from the impact point. At the end of the simulation time, a small part of the impactor mass is accumulated at the epicenter of the impact, compatibly with the experimental evidence. The equivalent plastic strain field predicted by the simulation is characterized by a peak of plastic deformation at around 2 millimeters from the epicenter. The calculated equivalent plastic strain field (Fig. 26 and Fig. 27) to the plastic strain extrapolated from the experimental micro-hardness values shows good adherence of the results with a slight overestimation of the peaks and a slight underestimation of the plastic strain field in the peripherical area.

The residual displacement of the plate is estimated as 3.6 millimeters (Fig. 25), about 22\% underestimated with respect to the average value of the displacements measured on the two experimental replicates (Fig. 10).
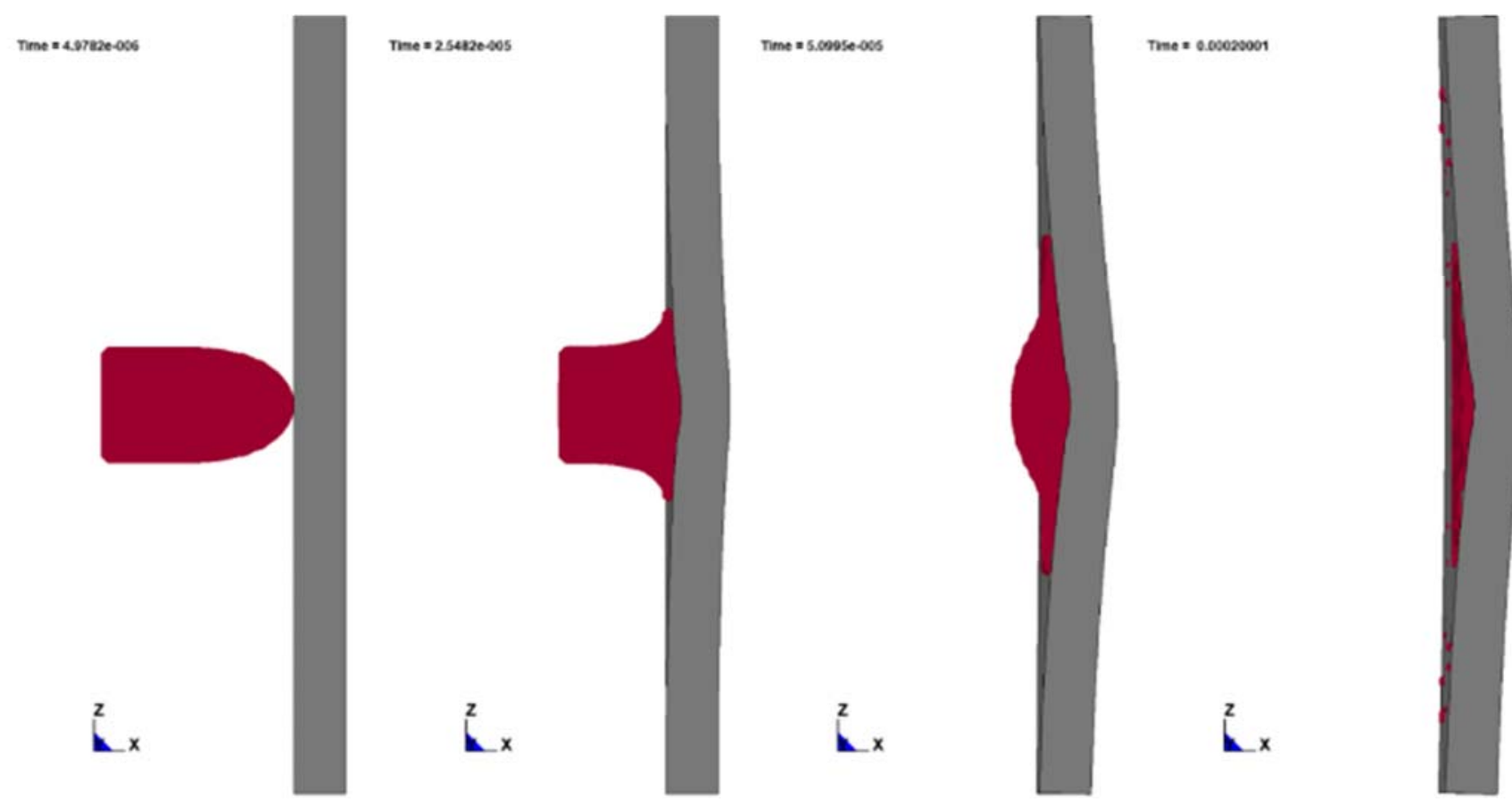

Figure 24: Dynamic interaction between bullet and plate. From left to right four frames showing the progressive deformation of the impactor and target from initial contact time at 0.0045 milliseconds to the stabilization of the plate at 0.2 milliseconds (time scale in seconds). 


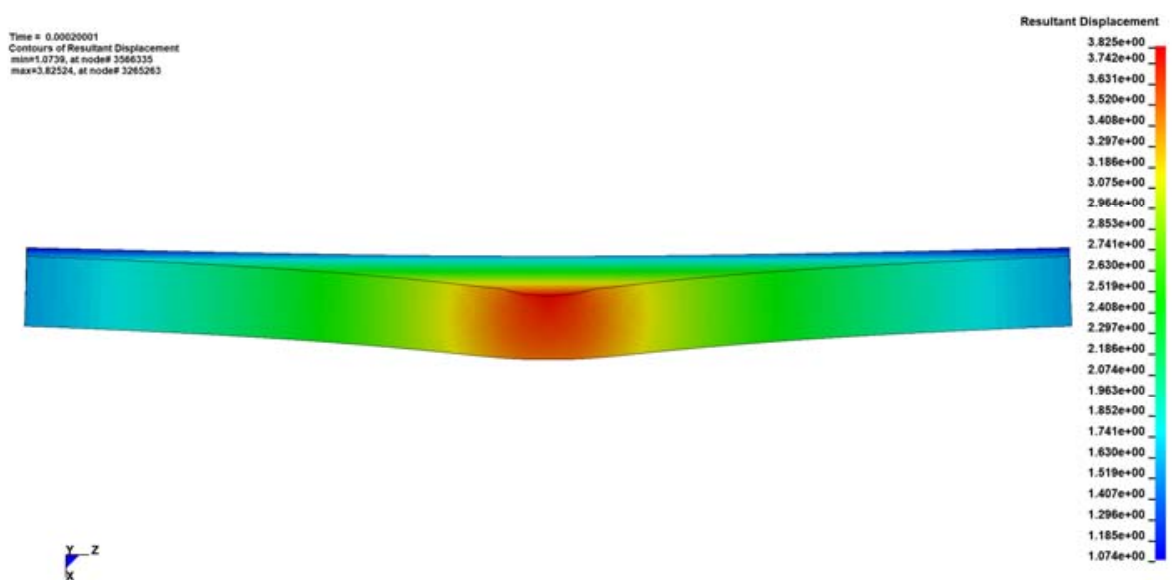

Figure 25: Field of residual resultant displacement of the plate at the end of the 90-degree impact.

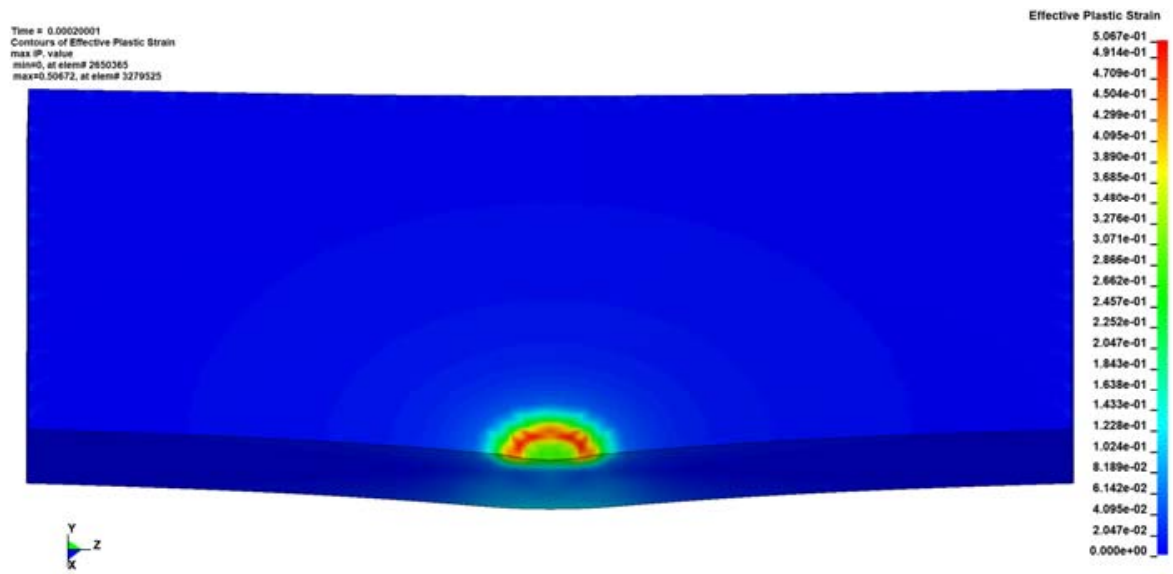

Figure 26: Field of equivalent plastic strain at the end of the 90-degree impact simulation.

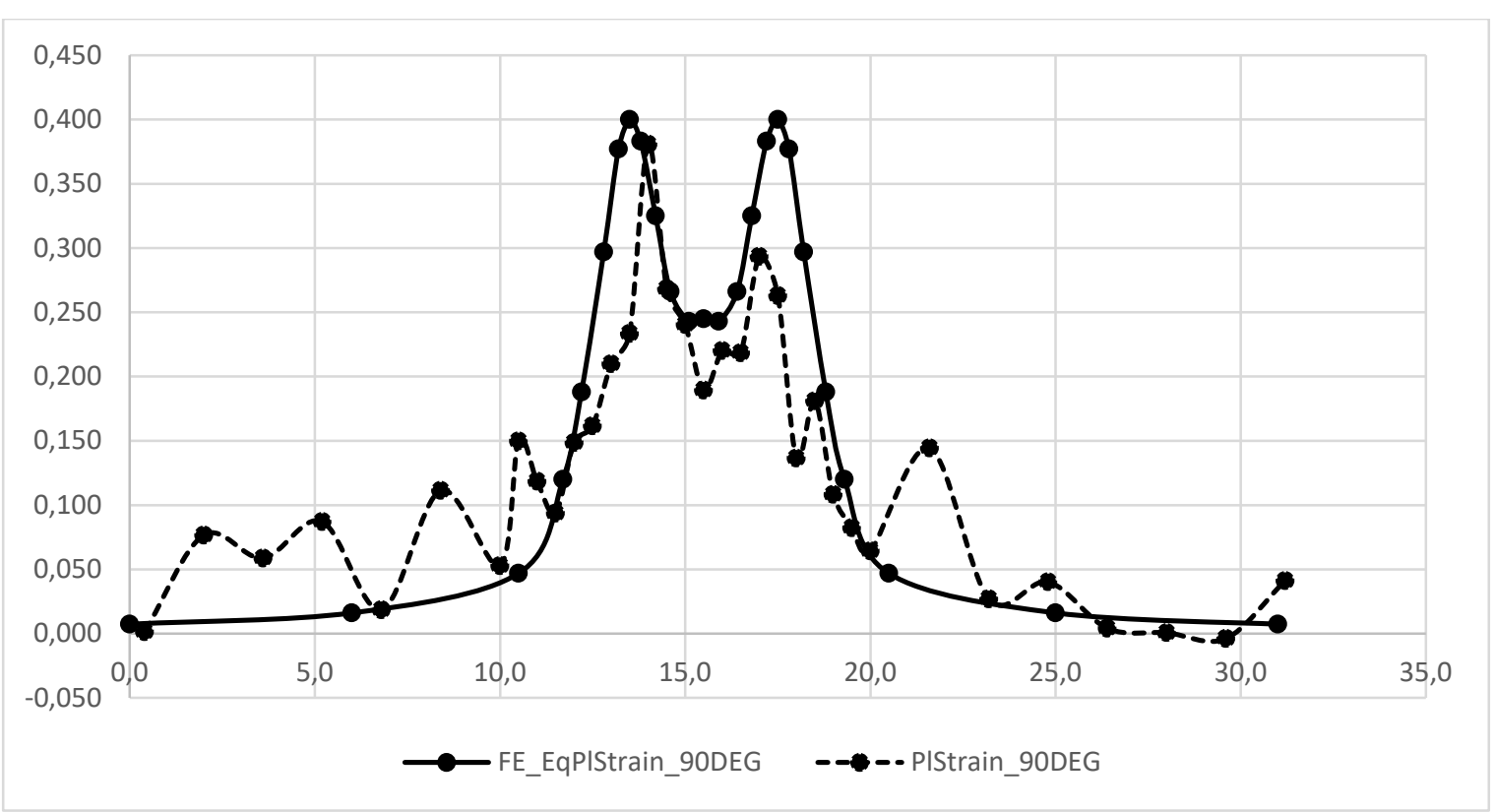

Figure 27: Equivalent plastic strain field at 0.2 millimeters beneath the impact surface as calculated by means of simulation (continuous line) compared with the plastic strain values calculated form micro-hardness measures by means of the linear correlation taken from Qiao et al. [13]. Abscissa values represent the coordinate along the section of the plate in millimeters. 


\section{5-degree impact}

Similarly, the simulation results of the 85-degree impact show an equivalent plastic strain show two peaks of plastic deformation at around 2 millimeters from the epicenter. The calculated equivalent plastic strain field (Fig. 30 and Fig. 31 ) compared to the plastic strain values extrapolated from the experimental micro-hardness profile shows good adherence of the results with an overestimation of the peaks and a slight underestimation of the plastic strain field in the peripherical area. The residual displacement of the plate (Fig. 29) is estimated as 3.5 millimeters, about 13\% underestimated with respect to the average value of the displacements measured on the two experimental replicates (Fig. 10).

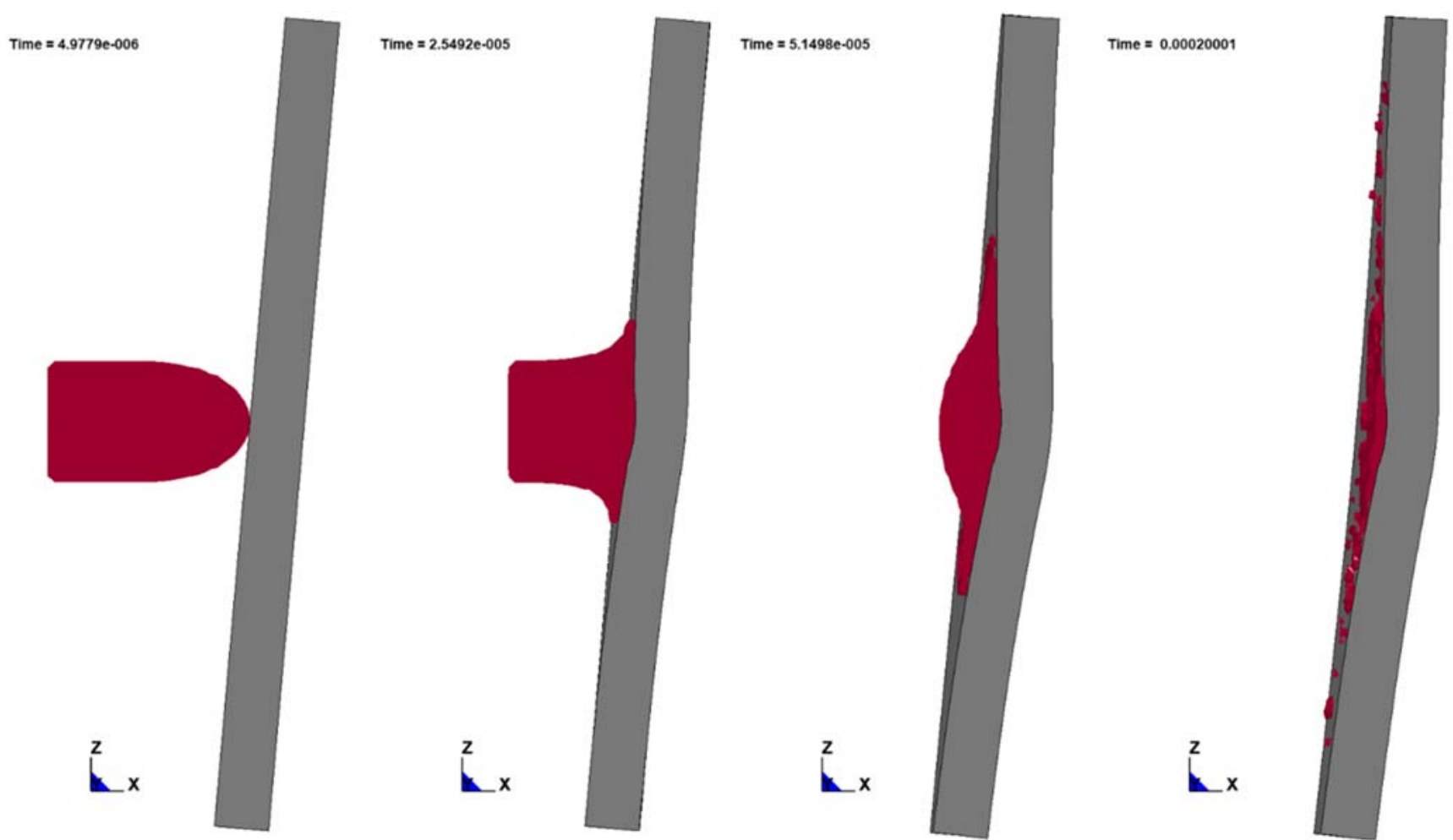

Figure 28: Dynamic interaction between bullet and plate. From left to right four frames showing the progressive deformation of the impactor and target from initial contact time at 0.005 milliseconds to the stabilization of the plate at 0.2 milliseconds (time scale in seconds).

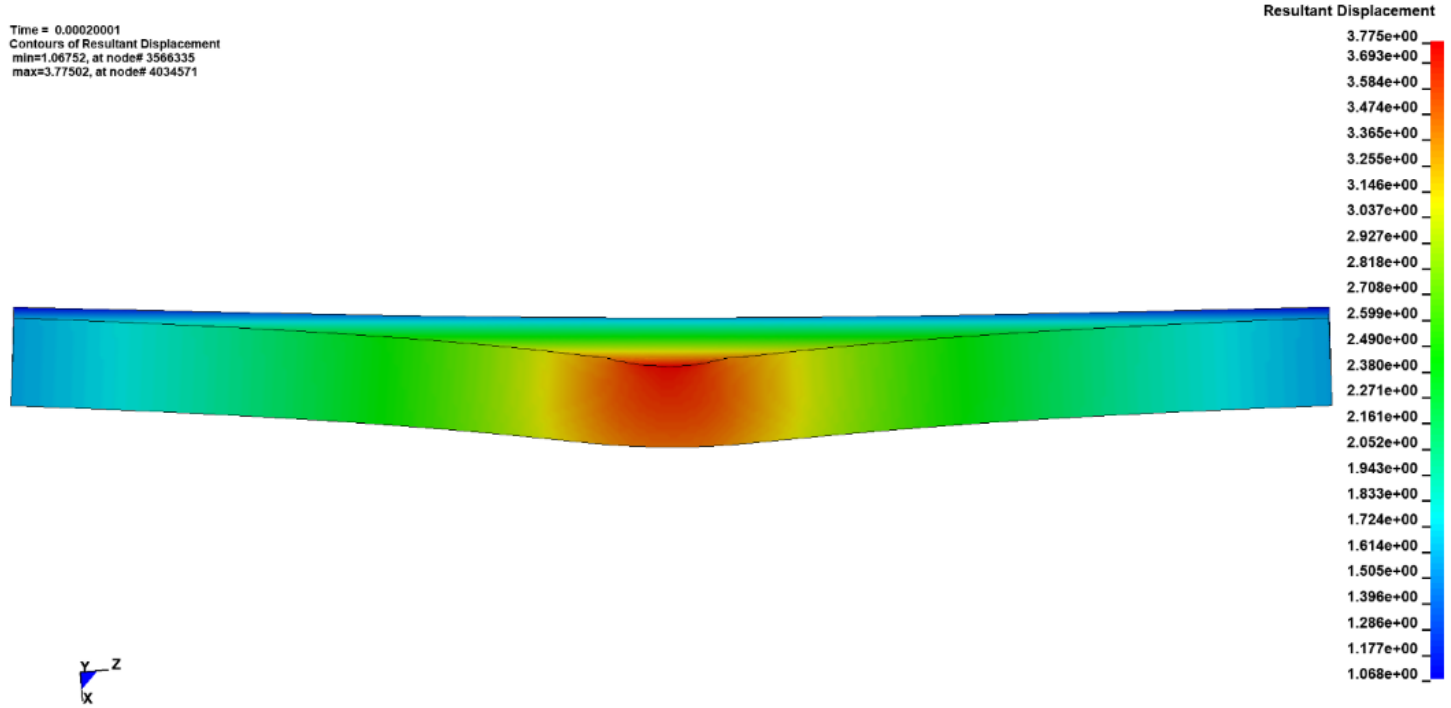

Figure 29: Field of residual resultant displacement of the plate at the end of the 85-degree impact. 


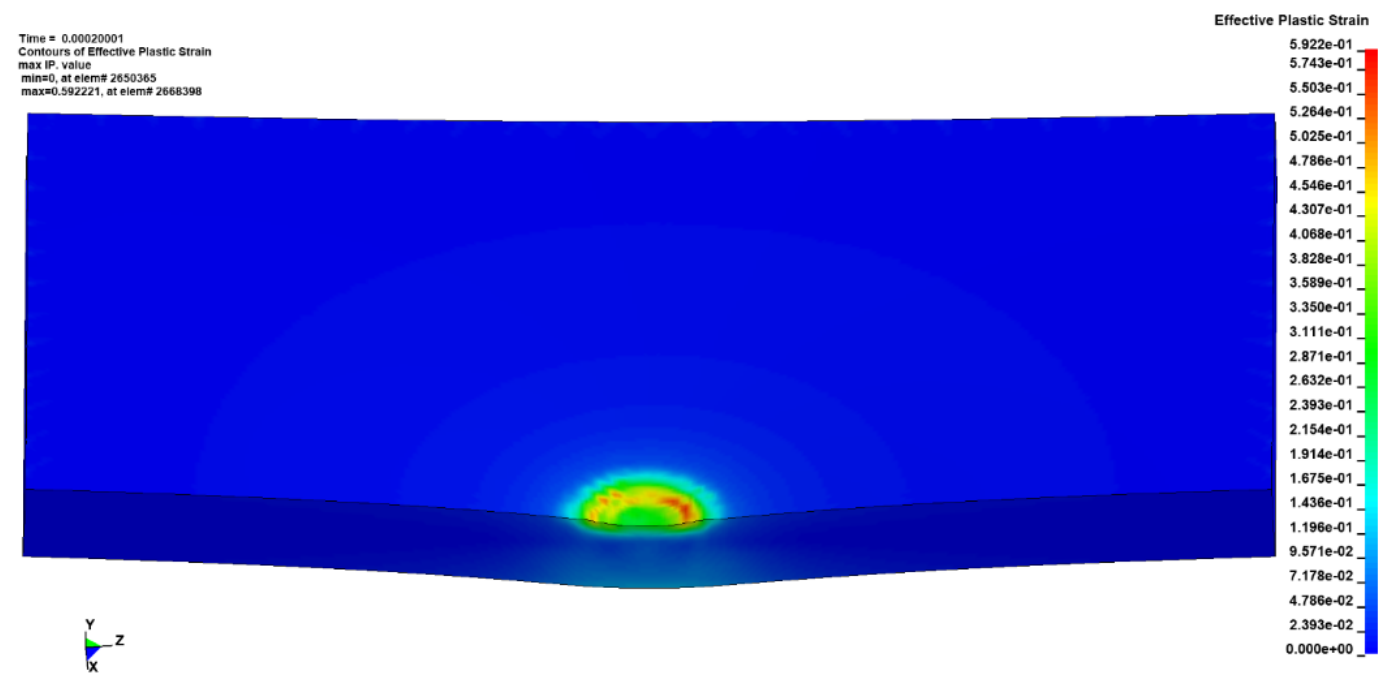

Figure 30: Field of equivalent plastic strain at the end of the 85-degree impact simulation.

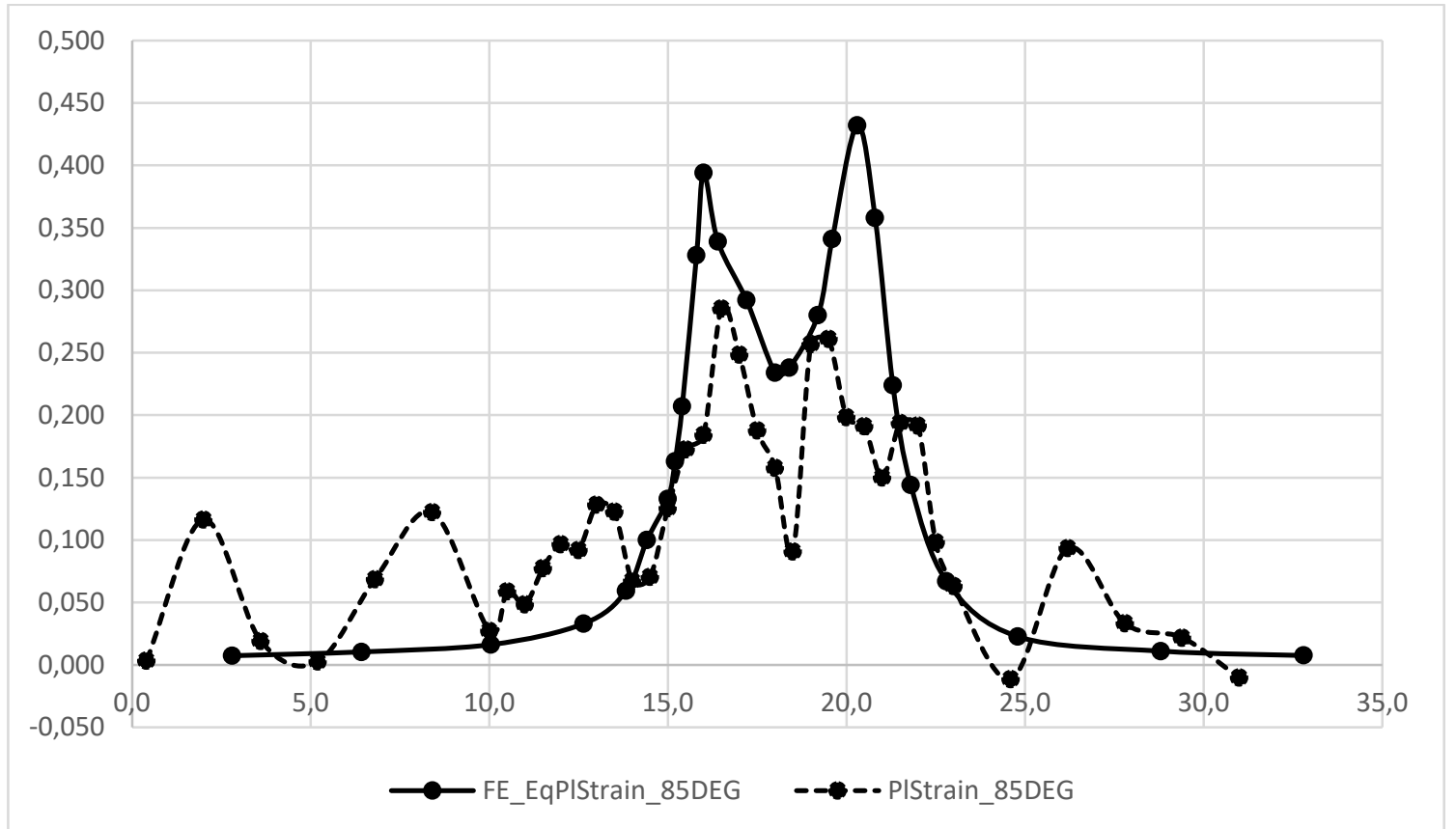

Figure 31: Equivalent plastic strain field at 0.2 millimeters beneath the impact surface as calculated by means of simulation (continuous line) compared with the plastic strain values calculated form micro-hardness measures by means of the linear correlation taken from Qiao et al. [13]. Abscissa values represent the coordinate along the section of the plate in millimeters.

\section{0-degree impact}

At 60-degree impact angle, the simulation still shows two peaks of plastic deformation at around 1.5 millimeters from the epicenter whereas the experimental curve appears smooth, without any significant peak, with maximum values around $23 \%$, i.e. about 30\% lower than the maximum peak estimated by the simulation (Fig. 34 and Fig. 35). Still, the simulation slightly underestimates the plastic deformation in the peripherical area.

The residual displacement of the plate (Fig. 33) is estimated as 2.6 millimeters, about 20\% underestimated with respect to the average value of the displacements measured on the two experimental replicates (Fig. 10). 

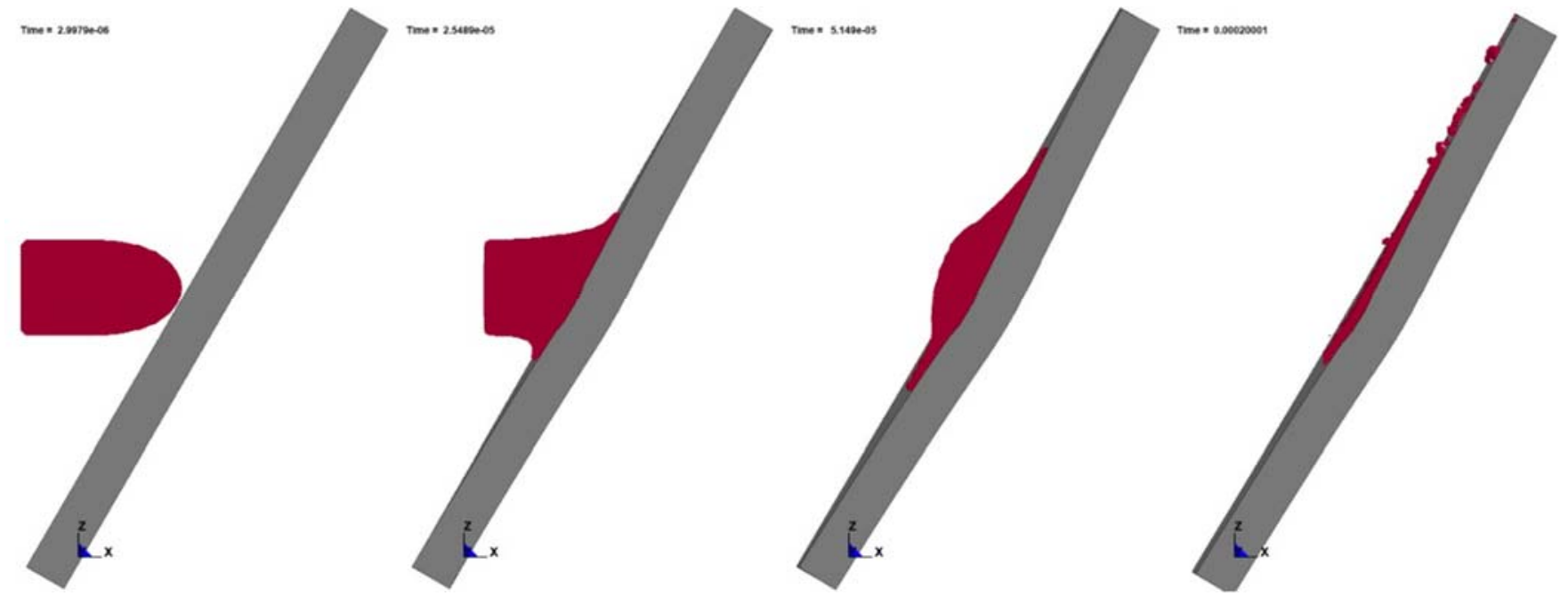

Figure 32: Dynamic interaction between bullet and plate. From left to right four frames showing the progressive deformation of the impactor and target from initial contact time at 0.003 milliseconds to the stabilization of the plate at 0.2 milliseconds (time scale in seconds).

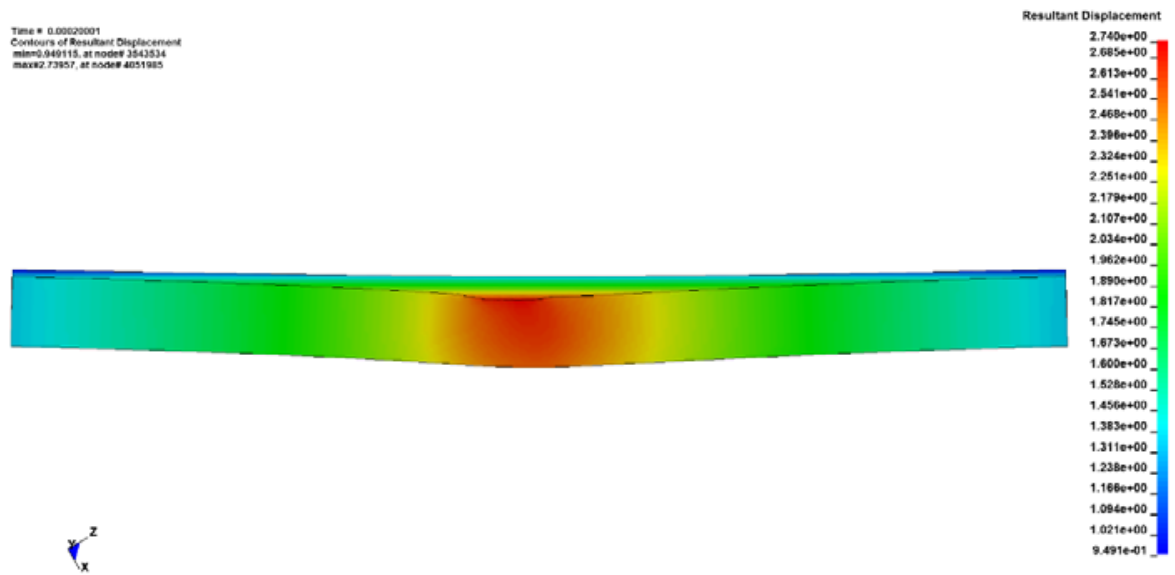

Figure 33: Field of residual resultant displacement of the plate at the end of the 60-degree impact.

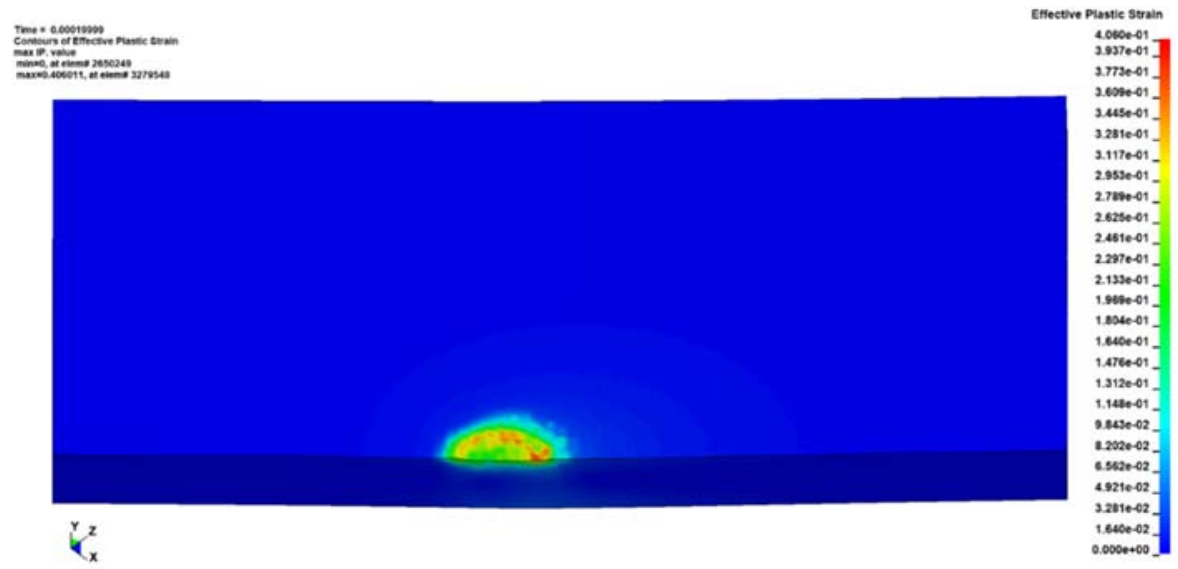

Figure 34: Field of equivalent plastic strain at the end of the 60-degree impact simulation. 


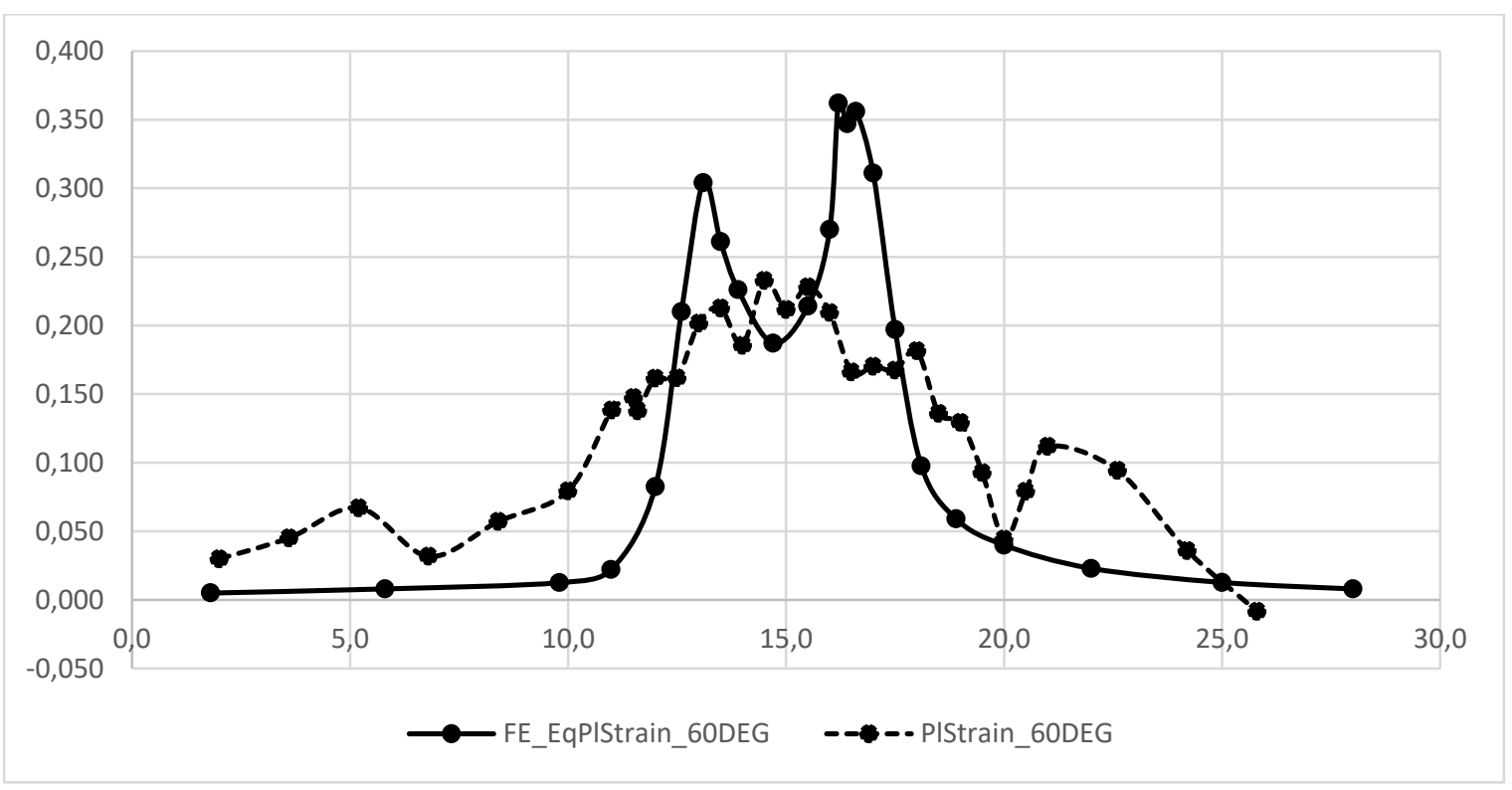

Figure 35: Equivalent plastic strain field at 0.2 millimeters beneath the impact surface as calculated by means of simulation (continuous line) compared with the plastic strain values calculated form micro-hardness measures by means of the linear correlation taken from Qiao et al. [13]. Abscissa values represent the coordinate along the section of the plate in millimeters.

\section{5-degree impact}

The 45-degree impact the simulation still predicts two peaks of plastic deformation at around 2.0 millimeters from the epicenter whereas the experimental curve is smooth without showing any significant peak. The maximum peak estimated by the simulation reaches $33.4 \%$, whereas the maximum experimental value is around $11.4 \%$. Thus, in the 45 -degree case, the plastic strain profile estimated by the simulation is much more severe than the real one in the surroundings of the epicenter but still underestimated in the peripherical area (Fig. 38 and Fig. 39).

The residual displacement of the plate is estimated as 1.6 millimeters (Fig. 37), about 8\% overestimated with respect to the average value of the displacements measured on the two sample replicates (Fig. 10).

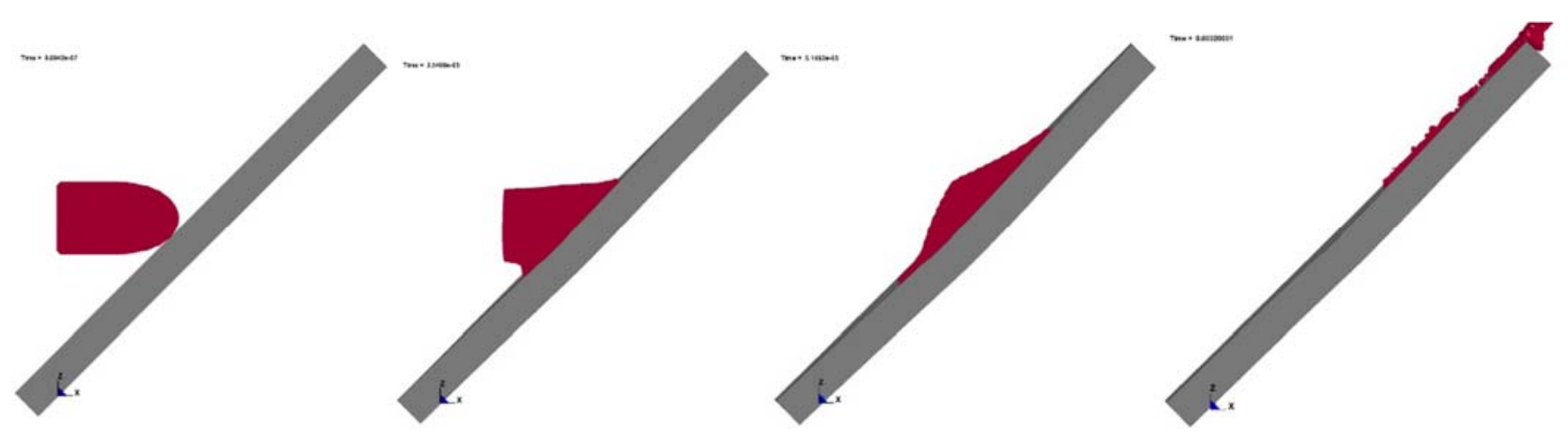

Figure 36: Dynamic interaction between bullet and plate. From left to right four frames showing the progressive deformation of the impactor and target from initial contact time at 0.003 milliseconds to the stabilization of the plate at 0.2 milliseconds (time scale in seconds).

\section{Spurious energy erosion and velocity correction}

The ALE formulation is characterized by a well-known intrinsic drawback, which consists in a spurious dissipation of the kinetic energy of the ALE material while encountering movements through the reference mesh[10]. This is due to the systematic error of the so-called advection algorithm that updates the velocity of the material moving through the ALE 
mesh. This causes the reduction of the available impact energy during the interaction phase between impactor and target as much as the material associated with the bullet encounters deformation. Anyway, this intrinsic problem can be easily quantified and overtook by analyzing the decay of the total energy during the interaction time of the impact, while the impactor transfers momentum to the target. For a soft impactor, the characteristic interaction time can be estimated as the time needed for the impactor to travel a distance equal to its length in the direction of movement. In the considered case the bullet is 15 millimeters long and travels at $322 \mathrm{~m} / \mathrm{s}$, therefore the interaction time is 46.6 microseconds. During this time, the total energy of the simulation encounters a reduction of $37 \%$ (Fig. 40), which is only due to the progressive reduction of velocity of the impactor during its deformation. The average kinetic energy available to the impactor during the interaction time is then reduced by $18.5 \%$.

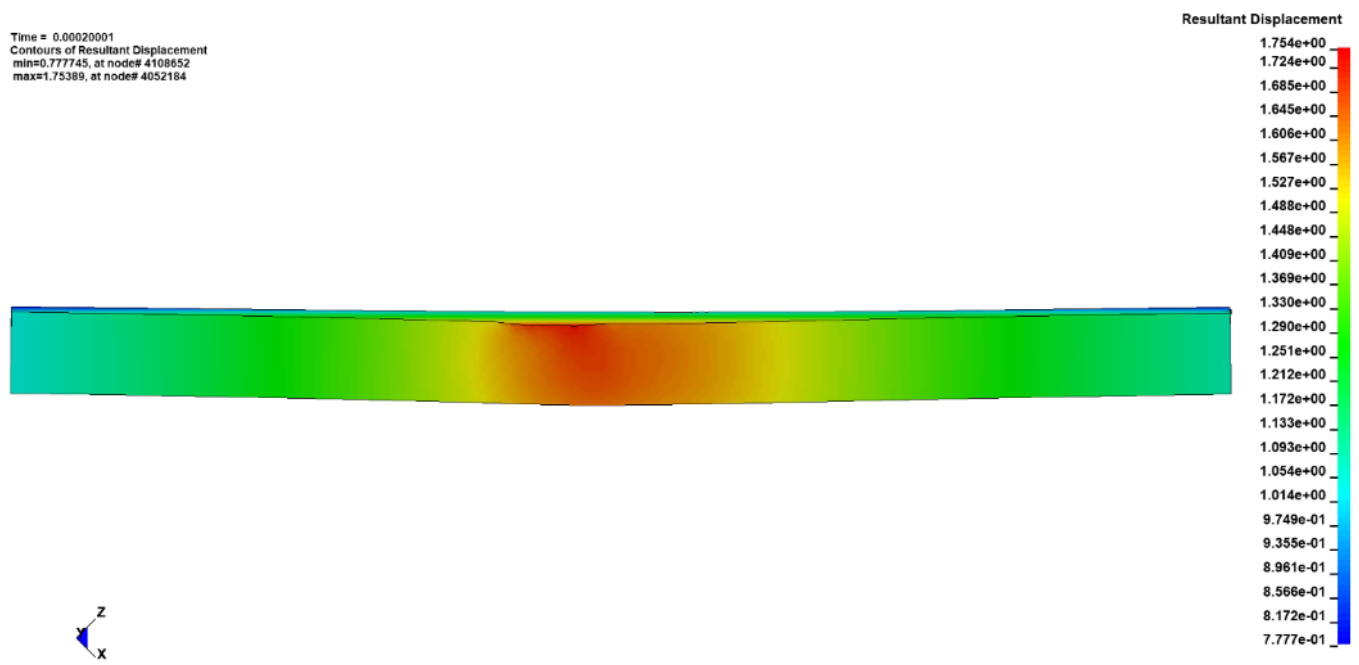

Figure 37: Field of residual resultant displacement of the plate at the end of the 60-degree impact.

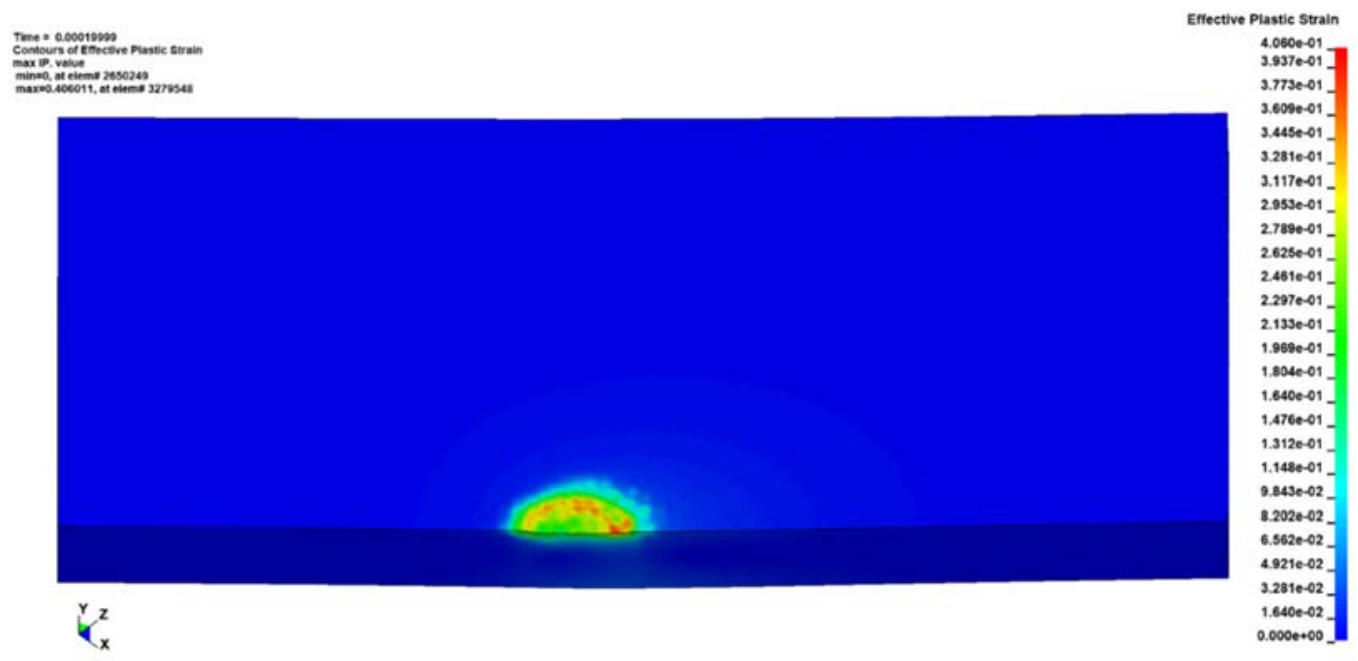

Figure 38: Field of equivalent plastic strain at the end of the 85-degree impact simulation.

Therefore, this phenomenon can be quantified and corrected by means of an increase of the initial speed of the impactor. In our case the initial energy must be increased by a factor $1 / 0.815=1.226$ which can be done by correcting the initial speed of the impactor by a factor 1.11. This way the energy effectively available to the impactor during the interaction phase will be correct even if slightly higher in the first half of the interaction phase and slightly lower in the second half. The results of the simulations with the corrected initial speed of the impactor show a clear improvement in the prediction of the residual displacements, as displayed in Fig. 41. 


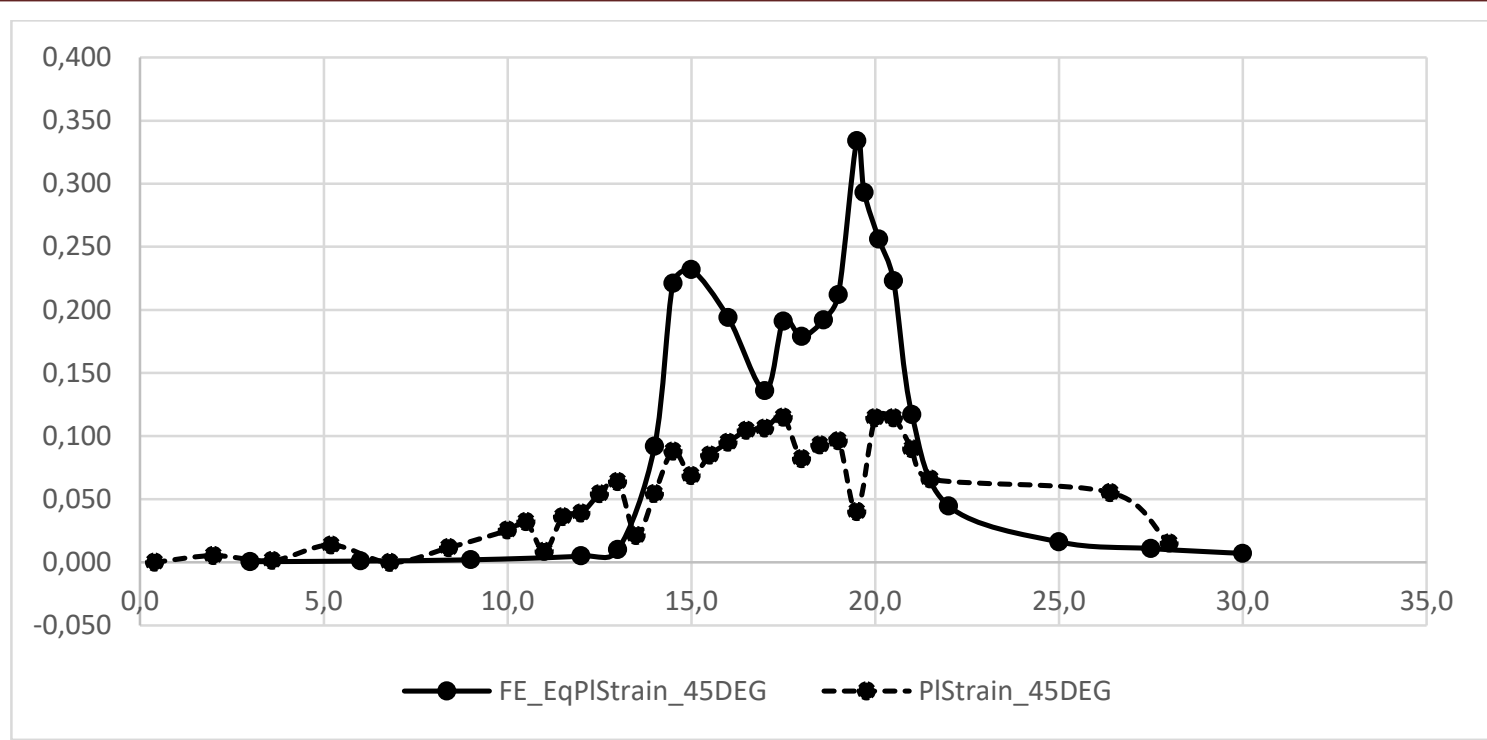

Figure 39: Equivalent plastic strain field at 0.2 millimeters beneath the impact surface as calculated by means of simulation (continuous line) compared with the plastic strain values calculated form micro-hardness measures by means of the linear correlation taken from Qiao et al. [13]. Abscissa values represent the coordinate along the section of the plate in millimeters.

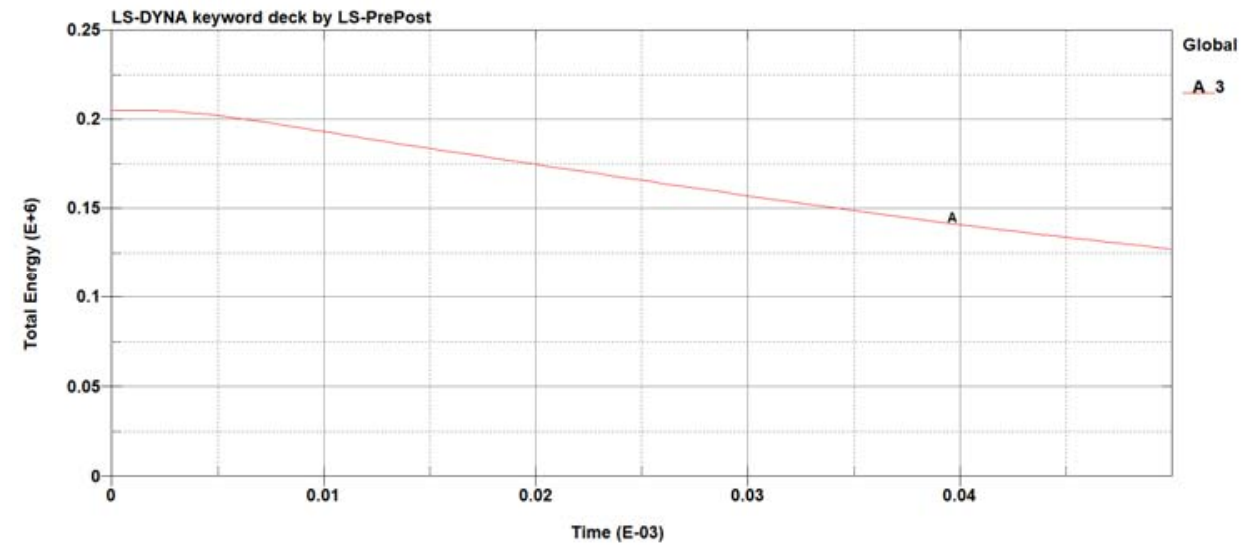

Figure 40: Decrease of the total energy of the simulation during the interaction time (in seconds).

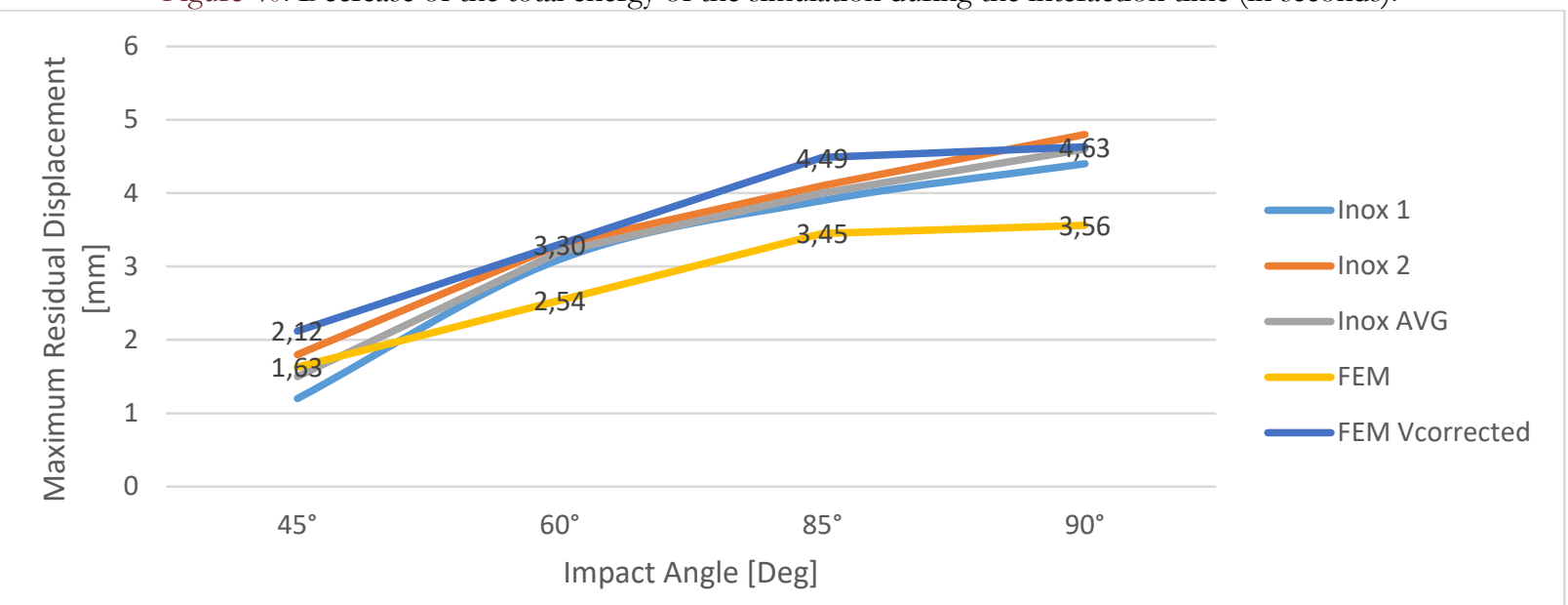

Figure 41: Comparison between experimental and numerical residual displacements. Light blue and orange lines represent the minimum and maximum experimental displacements. The grey line represents the average of the experimental results. The yellow line represents the results of the simulations without initial velocity correction. The dark blue line represents the simulation results with initial velocity corrected. 


\section{CONCLUSIONS}

$\mathrm{T}$ he study investigated the effects of bullet splash phenomena with the aim of developing and validating a simplified finite element model to be of practical use in the industry to simulate and optimize the response of protective structural systems subjected to splash bullet impacts.

The ballistic tests were conducted on 4-millimeter thick AISI 304L plates, shot by 9x21 FMJ bullets at various incidence angles $\left(90^{\circ}, 85^{\circ}, 60^{\circ}\right.$, and $\left.45^{\circ}\right)$. The visual analysis of the impact surface and the debris collection screen confirmed the complete fragmentation of the bullets with most of its mass being separated in small fragments and deflected by the target surface. According to the traces visible on the target and the number of holes reported by the collection screen, the number of fragments was estimated in the order of $10^{2}$. The residual displacements measured on the plates range from $4.6 \mathrm{~mm}$ at $90^{\circ}$ to $1.5 \mathrm{~mm}$ at $45^{\circ}$.

The micrographic analyses of the plates section near the impact epicenter show a smooth deformation field with no evident signs of grain distortion. To catch the plastic strain field across the section of the plates subjected to the impacts, forty microhardness measures were taken at $0.2 \mathrm{~mm}$ depth from the impact surface. The microhardness profiles show two different shapes depending on the acuteness of the impact angle. At $90^{\circ}$ and $85^{\circ}$ the microhardness distributions show an M-shaped profile, with of two peaks at a reciprocal distance of about $3 \mathrm{~mm}$, with a relative minimum in the middle, corresponding to the position of the epicenter of the impact. At $60^{\circ}$ and $45^{\circ}$ angles the microhardness profiles are smoother, like bell curves, with no evident peaks and the maximum value located at the epicenter of the impacts.

Based on these observations, the simplified numerical model was conceived on the assumption that the effects of the splashing impacts on the target are mostly due to the initial shape, inertia, and compressibility of the impactor, so that the impacts can be simulated as a fluid structure interaction, similarly to what is already being done in the industry to simulate 'soft' impacts like bird strikes or hail strikes. The simulation model was created based on the Arbitrary Lagrangian Eulerian (ALE) formulation, which allows to simulate very effectively the large deformation of the impactor with good stability of the calculation.

The results of the simulations in terms of plastic strain showed a good adhesion with the microhardness profiles of the plates. The simulation was able to predict the M-shaped plastic strain curve across the impact area, and the linear correlation between microhardness measures and plastic strain field allowed to quantitatively compare the predicted and experimental plastic strain fields, showing a very good prediction at $90^{\circ}$ and a progressively growing overestimation of the plastic strain peaks as the impact angles decrease. On the contrary, the simulation tends to slightly underestimate the effects of the impacts in the area exceeding the diameter of the bullet, where the samples show local peaks of plastic deformation due to the impacts and deflection of the fragments of the lead core and brass jacket.

The residual displacement estimated by the simulations compared to the measurements show an underestimation of the displacements filed of about $20 \%$ at higher angles. This is due to the spurious energy erosion intrinsic to the ALE formulation. This problem can be effectively solved by introducing a correction coefficient to the initial velocity of the bullet.

All the above confirm that the major simplifications introduced to model the bullet splash phenomenon are compatible with a reasonably accurate prediction of both local and global effects of the impacts.

Provided that the hypotheses of bullet splash are verified and the energy loss due to the advection algorithm is kept under control, the model can be effectively used in a conservative way to estimate the global effects of ballistic impacts on protective structural systems. The validation of the results shows that the model overestimates the local effects of the impacts at low angle, probably since the impactor is modelled as a mass of inviscid fluid, so it underestimates the actual energy dissipations due to the bullet fragmentation and deformation. Possible improvements and developments to the model can be put in place by testing a simplified pre-fragmented formulation to overcome the spurious energy loss and repeating the same experimentation with other types of real impactors and targets.

\section{ACKNOWLEDGEMENTS}

$\mathrm{T}$ his paper resumes some of the results of the research project started in 2016 and led by Marco V. Boniardi (Politecnico Di Milano) in collaboration with Reparto Investigazioni Scientifiche (RIS) of Carabinieri in Rome, Italy and Callens ${ }^{\circledR}$ AREA3. All the ballistic tests took place at RIS's shooting gallery in Rome and were conducted by the Carabinieri technicians. Riccardo Andreotti and Mauro Quercia were responsible for all the modelling and numerical simulation activities. Andrea Casaroli has conducted all the experimental, chemical, and metallurgical analyses. Riccardo 
Fossati collaborated to the experimental activities between 2016 and 2017 and developed his master's degree thesis on the subject.

\section{REFERENCES}

[1] Heimbs, S. (2011). Computational methods for bird strike simulations: A review, Comput. Struct., 89(23-24), pp. 2093112, DOI: $10.1016 /$ j.compstruc.2011.08.007.

[2] Anghileri, M., Castelletti, L.M.L., Invernizzi, F., Mascheroni, M. (2005). A survey of numerical models for hail impact analysis using explicit finite element codes, Int. J. Impact Eng., 31(8), pp. 929-44, DOI: 10.1016/j.ijimpeng.2004.06.009.

[3] Parker, S.P. (2003). Bullet Splash. Available at: https://encyclopedia2.thefreedictionary.com/bullet+splash. [accessed May 29, 2021].

[4] Edgerton, H.E., Jussim, E., Kayafas, G. (1987). Stopping Time: The Photographs of Harold Edgerton, New York, H.N. Abrams.

[5] Iqbal, M.A., Diwakar, A., Rajput, A., Gupta, N.K. (2012). Influence of projectile shape and incidence angle on the ballistic limit and failure mechanism of thick steel plates, Theor. Appl. Fract. Mech., 62(1), pp. 40-53, DOI: 10.1016/j.tafmec.2013.01.005.

[6] Yunfei, D., Wei, Z., Guanghui, Q., Gang, W., Yonggang, Y., Peng, H. (2014). The ballistic performance of metal plates subjected to impact by blunt-nosed projectiles of different strength, Mater. Des., 54, pp. 1056-1067, DOI: $10.1016 /$ j.matdes.2013.09.023.

[7] Camacho, G.T., Ortiz, M. (1996). Computational modelling of impact damage in brittle materials, Int. J. Solids Struct., 33(20-22), pp. 2899-2938, DOI: 10.1016/0020-7683(95)00255-3.

[8] Pandolfi, A., Ortiz, M. (2002). An efficient adaptive procedure for three-dimensional fragmentation simulations, Eng. Comput., 18(2), pp. 148-59, DOI: 10.1007/s003660200013.

[9] Bresciani, L.M., Manes, A., Romano, T.A., Iavarone, P., Giglio, M. (2016). Numerical modelling to reproduce fragmentation of a tungsten heavy alloy projectile impacting a ceramic tile: Adaptive solid mesh to the SPH technique and the cohesive law, Int. J. Impact Eng., 87, pp. 3-13, DOI: 10.1016/j.ijimpeng.2015.10.003.

[10] Day, J. (2009). Guidelines for ALE Modeling in LS-DYNA, .

[11] Smojver, I., Ivančević, D. (2011). Bird strike damage analysis in aircraft structures using Abaqus/Explicit and coupled Eulerian Lagrangian approach, Compos. Sci. Technol., 71(4), pp. 489-98, DOI: 10.1016/j.compscitech.2010.12.024.

[12] Gabrys, J., Schatz, J. (2004).The Use of LS-DYNA in the Columbia Accident Investigation and Return to Flight Activities. 8th International LS-DYNA Users Conference, Detroit, LSTC, pp. 1-10.

[13] Qiao, D., Zhang, W., Pan, T.Y., Crooker, P., David, S., Feng, Z. (2013). Evaluation of residual plastic strain distribution in dissimilar metal weld by hardness mapping, Sci. Technol. Weld. Join., 18(7), pp. 624-630, DOI: $10.1179 / 1362171813$ Y.0000000144.

[14] Gooch, G., Williams, M. (2014). Bullet Splash. Available at: https://www.oxfordreference.com/view/10.1093/oi/authority.20110803095534946. [accessed May 29, 2021].

[15] LSTC. (2015). LS-DYNA R8.0 Keyword User's Manual - Volume II - Material Models, vol. II.

[16] Gale, W.F., Totemeier, T.C. (2004).15 - Elastic properties, damping capacity and shape memory alloys. Smithells Metals Reference Book (Eighth Edition) Eighth Edition, Oxford, Butterworth-Heinemann, pp. 15-45.

[17] Nordberg, H. (2004). Note on the sensitivity of stainless steels to strain rate, AvestaPolarit Res. Found., 1(04), pp. 110. 